Research Article

\title{
Numerical Simulation and Experimental Analysis of Microstructure of Magnetorheological Fluid
}

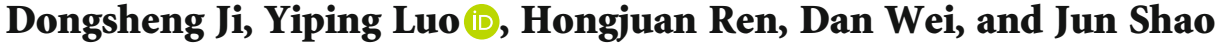 \\ School of Mechanical and Automotive Engineering, Shanghai University of Engineering Science, China \\ Correspondence should be addressed to Yiping Luo; lyp777@sina.com
}

Received 5 July 2019; Revised 13 September 2019; Accepted 16 October 2019; Published 14 November 2019

Academic Editor: Francesco Marotti de Sciarra

Copyright (C) 2019 Dongsheng Ji et al. This is an open access article distributed under the Creative Commons Attribution License, which permits unrestricted use, distribution, and reproduction in any medium, provided the original work is properly cited.

\begin{abstract}
Magnetorheological fluid is a new type of smart material that is sensitive to magnetic fields and has controllable performance. It is widely regarded for its unique magnetorheological effect and good rheological properties. For materials, the microstructure determines its macroscopic properties. In order to better study its macroscopic properties, it is necessary to have a more comprehensive understanding and deep understanding of its microstructure. In this paper, the magnetization process of magnetorheological fluid is analyzed from a microscopic point of view. Based on Newton's second law, the dynamic model of particle motion is established. The magnetic force, repulsive force, and viscous resistance of magnetic particles are analyzed. The finite difference numerical calculation method is used. The velocity-Verlet algorithm simulates the static microstructure chaining process of the magnetorheological fluid and the dynamic chaining process under shear force under different influencing factors. At the same time, a static observation device and a shear observation device were developed to observe the microstructure chaining morphology of magnetorheological fluid under different influencing factors, and to study the dynamic chaining law of magnetorheological fluid under the action of a shear force. Therefore, a reasonable contrast index is established, and the numerical simulation results are compared with the experimental observation results.
\end{abstract}

\section{Introduction}

With the rapid development of modern science and technology, people pay more and more attention to smart materials. The special properties of magnetorheological fluid (MRF) attract the attention of many countries and many scholars. MRF is a stable suspension [1,2], which is controlled by a magnetic field. It has a reversible reaction of milliseconds. This is also the fundamental reason why MRF produces MRF magnetorheological effect by forming a chain structure of magnetic particles under the action of a magnetic field. Therefore, only by studying the chaining process of magnetic particles under a magnetic field can we study the magnetorheological effect in essence.

Nowadays, many scientists have done a series of research on the chaining process of MRF particles. Shulman et al. [3] found that under the action of an external magnetic field, particles form elliptical polymers along the direction of the magnetic field and are arranged in chains from the microscopic structure of MRF. They approximated the equilibrium of the external and magnetic moments acting on the polymer by using knowledge of statistical physics, then obtained the shear stress of MRF. Lemaire et al. [4] also calculated from their own calculations the shear yield stress of MRF without demagnetization. Tang and Conrad [5] used the Maxwell stress tensor to mathematically simplify the flux linkage to a uniform plate and estimate the shear yield stress of the MRF. Rosenweig [6] also used the Maxwell stress tensor and proposed an average field continuous model based on the method of asymmetric stress analysis. It should be considered that under the action of a magnetic field, the particle chain may not be completely linear along the direction of the magnetic field. Peng and Li [7] proposed the assumption that the angle between the particle chain and the magnetic field obeys the normal distribution, and they proposed describing the shear strain. MRF shear force have expressions that affect the mechanical properties of MRF and other factors such as shear strain rate. Ciocanel et al. [8] considers the nonlinear magnetization of ferromagnetic particles in an external magnetic field. Considering the influence of magnetic force and 
fluid viscous resistance, the constitutive equation of MRF is established. Yi et al. [9] proposed a more precise dipole model by the same method and obtained the corresponding shear yield stress expression. Zhang et al. [10] proposed a Gaussian distribution model to study the properties of anisotropic magnetorheological elasticity, and they obtained the expression of magnetorheologically induced shear stress. The model improved the prediction of the properties of magnetorheological elastomers. Although some progress has been made in the above research, among the many models, there is no consensus that can accurately and systematically express the rheological properties of MRF. Therefore, the rheological properties of MRF need to be further studied.

In this paper, the chaining process of MRF is analyzed from the microscopic point of view. Firstly, the influence of particle size and particle volume fraction on the MRF microstructure is simulated by numerical simulation. At the same time, the static and dynamic shear observation devices developed by ourselves were used to observe the MRF samples and the influencing factors were analyzed. Finally, the numerical simulation results are compared with the experimental observations, and the microstructural chaining process of MRF can be effectively simulated, which has important guiding significance for the practical application of MRF.

\section{Simulation Calculation and Experiment}

2.1. Numerical Simulation Method. At present, numerical simulation can simulate the microstructure of MRF using multiple variables and is closer to the real model. Among them, molecular dynamics simulation is one of the most important methods for studying the microstructure of MRF. The numerical calculation based on the finite difference method is commonly used, and the velocity-Verlet algorithm is adopted in this paper. In the simulation, the initial position of particles is randomly generated by a computer, the initial velocity is zero, and the simulation time step is $\Delta t$. The calculation formulas of the algorithm are as follows:

(1) Location of $t+\Delta t$ moment

$$
r(t+\Delta t)=r(t)+v(t) \cdot \Delta t+\frac{1}{2} a(t) \Delta t^{2}
$$

(2) Speed of $t+\Delta t$ hours

$$
v(t+\Delta t)=v(t)+\frac{1}{2}[a(t)+a(t+\Delta t)] \Delta t
$$

Formula (1) and formula (2) are solved by the iteration program written in MATLAB, and the iteration is terminated after the number of iterations. In the process of numerical simulation, it takes a lot of time to simulate a complete iteration, especially when calculating the force between particles, because the force of a particle needs to consider the influence

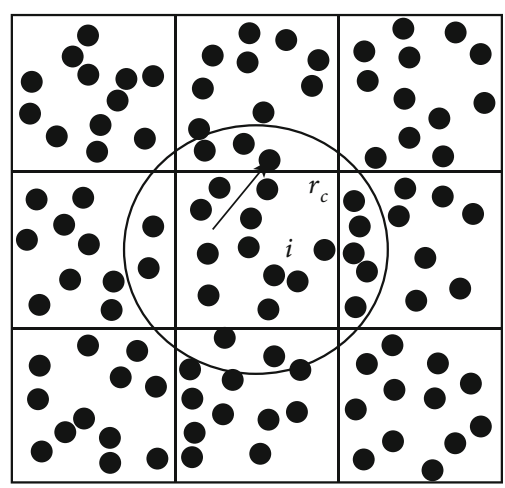

FIgURE 1: Schematic diagram of a method combining the cell list method with the Verlet list method.

of other particles in the system on the particle. For an MRF system with $N$ particles, if an efficient algorithm is not used, it is necessary to calculate the interaction between the $N(N-1) / 2$ pairs of particles in each incremental step, the force acting on the particles. The calculation time is proportional to $N^{2}$. The Verlet list method and the cell list method can accelerate the calculation of the force, so that the calculation time is proportional to $N$. The Verlet list method calculates the interaction force of magnetic particles in $N$ order of magnitude, but updates the Verlet list in $N^{2}$ order of magnitude. The time of the cell list method in calculating the interaction force of the magnetic particles and the time to update the cell list are all $N$ orders of magnitude. However, the Verlet listing method has a higher ratio of effective adjacent particle pairs than a cell listing method. Therefore, the Verlet listing method and the cell listing method are combined, as shown in Figure 1.

When there is no external magnetic field, the magnetic particles are randomly distributed in the carrier liquid, and the initial velocity is zero. Once the magnetic field is applied, the magnetic particles are instantly magnetized into dipoles, which are combined by magnetic force and resistance. Therefore, it has a certain acceleration, so the speed increment and the displacement increment can be obtained immediately, and the distribution position of the magnetic particles will also change, thereby causing the change of the force and the acceleration, and sequentially obtaining the acceleration in each time step, velocity, and displacement; when these physical quantities no longer change, the chaining process of the magnetic particles can be completed, which is the entire simulation process.

2.2. Force Analysis of Magnetic Particles. From the microscopic observation in Figure 2, it can be seen that the magnetic particles are randomly dispersed in the carrier fluid without an external magnetic field. Once a certain magnetic field is applied to it, the magnetic particles are magnetized into magnetic dipoles and gradually arranged into chains under the action of magnetic force. At this time, the MRF viscosity increases, the shear yield stress increases, and the MRF gradually changes from the original fluid state to the solid 


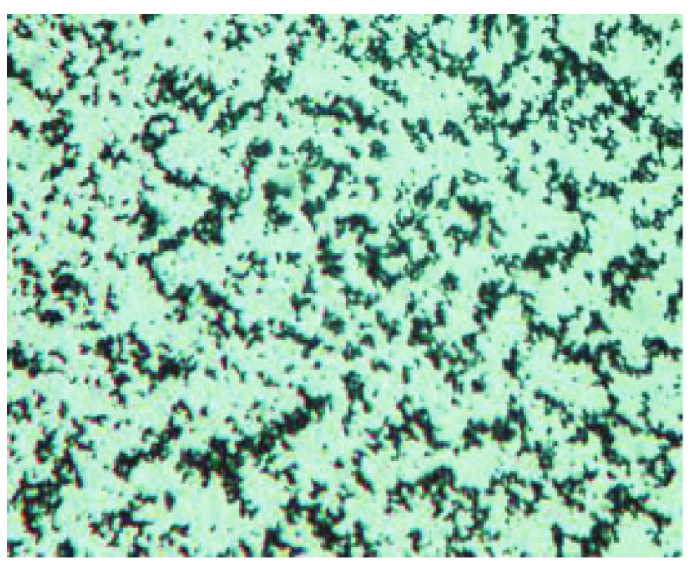

(a) No magnetic field

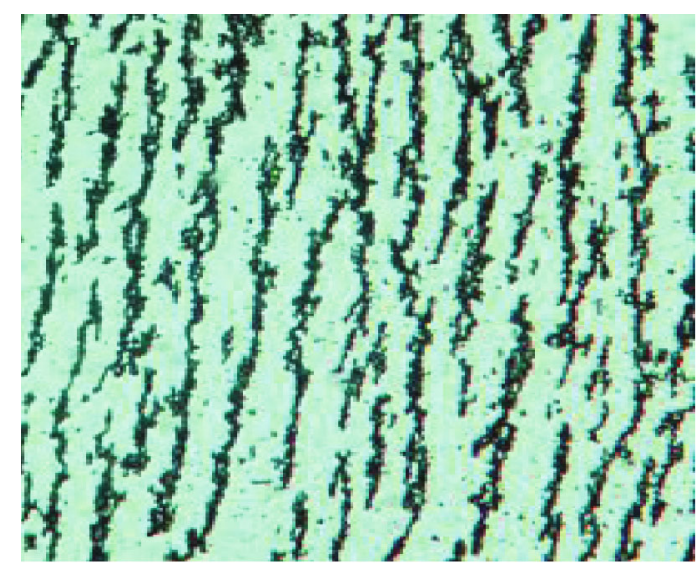

(b) External magnetic field

FIGURE 2: State diagram of MRF in the presence of a magnetic field and no magnetic field.

state. When the applied magnetic field increases to a certain value, all the magnetic dipole magnetizations become saturated and neatly arranged along the direction of the applied magnetic field; at this time MRF reaches the maximum shear yield stress value, and MRF completely transforms into a solid-like state. According to the magnetic dipole theory [11], there are two magnetic particles. Magnetic particles have two magnetic poles: $Q_{m}^{+}$and $Q_{m}^{-}$.

The magnetization is a physical quantity describing the magnetization direction and the degree of magnetization of the magnetic medium, and it represents the vector sum of magnetic moments of the magnetic dipole in the magnetic medium per unit volume. If any voxel $\Delta V$ is taken in the magnetic medium, assuming that there are a large number of magnetic dipoles in the voxel and the magnetic moment of each dipole is $m$, the magnetization expression is

$$
M=\frac{\sum m}{\Delta V}
$$

If the volume of the magnetic particles is small, the orientation of the magnetic domains inside the particles can be approximated. In this case, the particles can be regarded as a magnetic dipole alone, and the magnetic moment of the magnetic particles is $m$ and the volume is $V$. In formula $((3))$, the magnetic moment of any magnetic particle is

$$
M=V M \text {. }
$$

This is shown in Figure 3. According to Coulomb's law, the magnetic field generated by the magnetic pole at $r$ is $\tilde{\mathbf{H}}$ :

$$
\tilde{\mathbf{H}}=\frac{1}{4 \pi \mu_{0}} \frac{Q_{m}}{r^{2}} \widehat{\mathbf{r}}
$$

In formula (5), the unit vector is in the $\widehat{\mathbf{r}}-\mathbf{r}$ direction.

A magnetic dipole has two magnetic poles, so the magnetic field generated by the magnetic dipole at point $P$ should

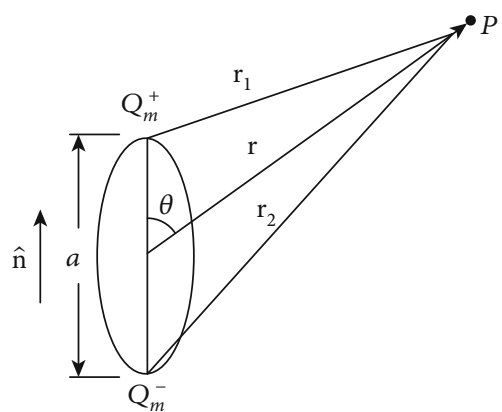

FIgURE 3: Schematic diagram of the magnetic field strength generated by the magnetic dipole at point $P$.

be the sum of the magnetic fields generated by the two magnetic poles:

$$
\tilde{\mathbf{H}}=\frac{Q_{m}}{4 \pi \mu_{0}}\left(\frac{\widehat{\mathbf{r}}_{\mathbf{1}}}{r_{1}^{2}}-\frac{\widehat{\mathbf{r}}_{\mathbf{2}}}{r_{2}^{2}}\right) .
$$

In the formula, $\widehat{\mathbf{r}}_{1}$ and $\widehat{\mathbf{r}}_{2}$ are two pole-to-point displacement vectors and $\widehat{\mathbf{n}}$ is the unit vector of the direction of the applied magnetic field, where $\mathbf{a}=a \widehat{\mathbf{n}}$.

As can be seen from Figure 3,

$$
\begin{aligned}
& \mathbf{r}_{1}=\mathbf{r}-\frac{1}{2} \mathbf{a} \\
& \mathbf{r}_{2}=\mathbf{r}+\frac{1}{2} \mathbf{a} .
\end{aligned}
$$

Using the unit vector, equation (7) becomes

$$
\begin{aligned}
& \widehat{\mathbf{r}}_{1}=\frac{\mathbf{r}_{1}}{r_{1}}=\frac{1}{r_{1}}\left(\mathbf{r}-\frac{1}{2} \mathbf{a}\right), \\
& \widehat{\mathbf{r}}_{2}=\frac{\mathbf{r}_{2}}{r_{2}}=\frac{1}{r_{2}}\left(\mathbf{r}+\frac{1}{2} \mathbf{a}\right) .
\end{aligned}
$$


According to the cosine theorem,

$$
\begin{aligned}
& r_{1}^{2}=r^{2}+\left(\frac{a}{2}\right)^{2}-r a \cos \theta \\
& r_{2}^{2}=r^{2}+\left(\frac{a}{2}\right)^{2}+r a \cos \theta
\end{aligned}
$$

In the formula (6),

$$
\begin{aligned}
\frac{\widehat{\mathbf{r}}_{1}}{r_{1}^{2}}-\frac{\widehat{\mathbf{r}}_{2}}{r_{2}^{2}} & =\left[\frac{1}{r_{1}^{3}}\left(\mathbf{r}-\frac{1}{2} \mathbf{a}\right)-\frac{1}{r_{2}^{3}}\left(\mathbf{r}+\frac{1}{2} \mathbf{a}\right)\right] \\
& =\left[\frac{r_{2}^{3}-r_{1}^{3}}{r_{1}^{3} r_{2}^{3}} \mathbf{r}-\frac{r_{1}^{3}+r_{2}^{3}}{2 r_{1}^{3} r_{2}^{3}} \mathbf{a}\right] .
\end{aligned}
$$
obtain

By substituting formula (10) into formula (6), one can

$$
\tilde{\mathbf{H}}=\frac{Q_{m}}{4 \pi \mu_{0}}\left(\frac{r_{2}^{3}-r_{1}^{3}}{r_{1}^{3} r_{2}^{3}} \mathbf{r}-\frac{r_{1}^{3}+r_{2}^{3}}{2 r_{1}^{3} r_{2}^{3}} \mathbf{a}\right)
$$

According to the definition of magnetic dipole moment $j$ and the relationship between magnetic moment and magnetic dipole moment

$$
\begin{aligned}
& \mathbf{j}=Q_{m} \mathbf{a}, \\
& \mathbf{j}=\mu_{0} \mathbf{m} .
\end{aligned}
$$

It can be seen from formula (12) that the magnetic field strength generated by any one of the magnetic dipoles of $Q_{m} / \mu_{0}=\mathbf{m} / \mathbf{a}$ and substituted into formula (11) is

$$
\tilde{\mathbf{H}}=\frac{m}{4 \pi a}\left(\frac{r_{2}^{3}-r_{1}^{3}}{r_{1}^{3} r_{2}^{3}} \mathbf{r}-\frac{r_{1}^{3}+r_{2}^{3}}{2 r_{1}^{3} r_{2}^{3}} \mathbf{a}\right)
$$

where $m$ is the Euclidean norm of $\mathbf{m}$ magnetic moment, that is, $m=\|\mathbf{m}\|$.

In free space, the magnetic field strength $\tilde{\mathbf{H}}$ and the magnetic induction $\tilde{\mathbf{B}}$ satisfy the following relationship:

$$
\tilde{\mathbf{B}}=\mu_{0} \tilde{\mathbf{H}}
$$

Therefore, the magnetic induction produced by a magnetic dipole is

$$
\tilde{\mathbf{B}}=\mu_{0} \tilde{\mathbf{H}}=\frac{\mu_{0} m}{4 \pi a}\left(\frac{r_{2}^{3}-r_{1}^{3}}{r_{1}^{3} r_{2}^{3}} \mathbf{r}-\frac{r_{1}^{3}+r_{2}^{3}}{2 r_{1}^{3} r_{2}^{3}} \mathbf{a}\right)
$$

From equation (15), it is known that the magnetic induction intensity of any magnetic dipole $j$ relative to another magnetic dipole $i$ is

$$
\tilde{\mathbf{B}}_{i j}=\frac{\mu_{0} m_{j}}{4 \pi a}\left(\frac{r_{2}^{3}-r_{1}^{3}}{r_{1}^{3} r_{2}^{3}} \mathbf{r}_{i j}-\frac{r_{1}^{3}+r_{2}^{3}}{2 r_{1}^{3} r_{2}^{3}} \mathbf{a}\right)
$$

In formula (16), $a$ is the diameter of dipole particles, where $a=2 R ; r_{i j}$ is the relative position of the dipole particle $j$ to the dipole particle $i$; and $\mu_{0}$ is vacuum permeability.

From the cosine theorem, we can see that in equation (16),

$$
\begin{aligned}
& r_{1}=\sqrt{r_{i j}^{2}+R^{2}-2 R r_{i j} \cos \theta}, \\
& r_{2}=\sqrt{r_{i j}^{2}+R^{2}+2 R r_{i j} \cos \theta} .
\end{aligned}
$$

Under the action of a uniform magnetic field $\mathbf{B}_{0}$, the force $\mathbf{F}_{i}$ of any magnetic dipole $i$ in the magnetic field is

$$
\mathbf{F}_{i}=-\mathbf{m} \cdot \nabla\left(\mathbf{B}_{0}+\tilde{\mathbf{B}}_{i}\right)-\mathbf{m} \cdot \nabla\left(\mathbf{B}_{0}+\sum_{j \neq i} \tilde{\mathbf{B}}_{i j}\right)=-\mathbf{m} \cdot \nabla \sum_{j \neq i} \tilde{\mathbf{B}}_{i j} .
$$

In formula (18), $\mathbf{B}_{0}$ adds a uniform magnetic field, so $\nabla \mathbf{B}_{0}=0 ; \sum_{j \neq i} \tilde{\mathbf{B}}_{i j}$ is the sum of the magnetic intensities generated by the other dipoles $j$ at the magnetic dipole $i$.

Substituting formula (17) into formula (18), we have

$$
\begin{aligned}
\mathbf{F}_{i} & =\frac{\mu_{0}}{8 \pi} \sum_{j \neq i} \frac{m}{R} \mathbf{m} \cdot \mathbf{i}_{k} \frac{\partial}{\partial x_{k}}\left\{\frac{\mathbf{r}_{i j}-R \widehat{\mathbf{n}}}{\left[r_{i j}^{2}+R^{2}-2 R r_{i j} \cos \theta\right]^{3 / 2}}-\frac{\mathbf{r}_{i j}+R \widehat{\mathbf{n}}}{\left[r_{i j}^{2}+R^{2}+2 R r_{i j} \cos \theta\right]^{3 / 2}}\right\} \\
& =\frac{\mu_{0}}{8 \pi} \sum_{j \neq i} \frac{m}{R}\left\{\frac{\mathbf{m}}{r_{1}^{3}}-\frac{m\left(3 r_{i j} \cos \theta-3 R\right)\left(\mathbf{r}_{i j}-R \widehat{\mathbf{n}}\right)}{r_{1}^{5}}-\frac{\mathbf{m}}{r_{2}^{3}}+\frac{m\left(3 r_{i j} \cos \theta+3 R\right)\left(\mathbf{r}_{i j}+R \widehat{\mathbf{n}}\right)}{r_{2}^{5}}\right\} \\
& =\frac{3 \mu_{0}}{8 \pi} \sum_{j \neq i} \frac{m^{2}}{R r_{1}^{5} r_{2}^{5}}\left\{\left[r_{i j} \cos \theta\left(r_{1}^{5}-r_{2}^{5}\right)+R\left(r_{1}^{5}+r_{2}^{5}\right)\right] \mathbf{r}_{i j}\right. \\
& \left.+\left[\frac{1}{3}\left(r_{1}^{2} r_{2}^{5}-r_{1}^{5} r_{2}^{2}\right)+R r_{i j} \cos \theta\left(r_{1}^{5}+r_{2}^{5}\right)+R^{2}\left(r_{1}^{5}-r_{2}^{5}\right)\right] \widehat{\mathbf{n}}\right\} .
\end{aligned}
$$


Hypothesis $\left\|\mathbf{r}_{1}\right\| \gg a,\left\|\mathbf{r}_{2}\right\| \gg a$ : there is $a /\left\|\mathbf{r}_{1}\right\| \approx 0$, $a /\left\|\mathbf{r}_{2}\right\| \approx 0$, that is, $r_{1} \approx r-(a / 2) \cos \theta, r_{2} \approx r+(a / 2) \cos \theta$. In the substitution type of (15), the magnetic induction generated by a magnetic dipole is

$$
\tilde{\mathbf{B}}=\frac{\mu_{0}}{4 \pi r^{3}}[3(\mathbf{m} \cdot \widehat{\mathbf{r}}) \widehat{\mathbf{r}}-\mathbf{m}] .
$$

Similarly, the magnetic induction of any magnetic dipole $j$ relative to another magnetic dipole $i$ can be simplified as

$$
\tilde{\mathbf{B}}_{i j}=\frac{\mu_{0}}{4 \pi r^{3}}\left[3\left(\mathbf{m}_{j} \cdot \widehat{\mathbf{r}}_{i j}\right) \widehat{\mathbf{r}}_{i j}-\mathbf{m}_{j}\right] .
$$

Substituting formula (21) into formula (19), the magnetic interaction force that any magnetic dipole $W$ is subjected to is simplified as follows:

$$
\begin{aligned}
\mathbf{F}_{i} & =\sum_{j \neq i} \frac{3 \mu_{0} m^{2}}{16 \pi a}\left\{\left[4 r_{i j} \cos \theta\left(\frac{1}{r_{2}^{5}}-\frac{1}{r_{1}^{5}}\right)+2 a\left(\frac{1}{r_{2}^{5}}+\frac{1}{r_{1}^{5}}\right)\right] \mathbf{r}_{i j}\right\} \\
& +\left[2 r_{i j} a \cos \theta\left(\frac{1}{r_{2}^{5}}+\frac{1}{r_{1}^{5}}\right)+a^{2}\left(\frac{1}{r_{2}^{5}}-\frac{1}{r_{1}^{5}}\right)+\frac{4}{3}\left(\frac{1}{r_{1}^{3}}-\frac{1}{r_{2}^{3}}\right)\right] \widehat{\mathbf{n}} \\
& =\sum_{j \neq i} \frac{3 \mu_{0} m^{2}}{16 \pi a}\left\{\left[4 r_{i j} \cos \theta\left(-\frac{5}{r_{i j}^{6}} a \cos \theta\right)+2 a \frac{2}{r_{i j}^{5}}\right] \mathbf{r}_{i j}\right\} \\
& +\left[2 r_{i j} a \cos \theta \frac{2}{r_{i j}^{5}}+a^{2}\left(-\frac{5}{r_{i j}^{6}} a \cos \theta\right)+\frac{4}{3} \frac{3}{r_{i j}^{4}} a \cos \theta\right] \widehat{\mathbf{n}} \\
& =\sum_{j \neq i} \frac{3 \mu_{0} m^{2}}{4 \pi r_{i j}^{4}}\left[\left(1-5 \cos ^{2} \theta\right) \widehat{\mathbf{r}}+\left(2 \cos \theta-\frac{5 a^{2}}{4 r_{i j}^{2}} \cos \theta\right) \widehat{\mathbf{n}}\right] \\
& =\sum_{j \neq i} \frac{3 \mu_{0} m^{2}}{4 \pi r_{i j}^{4}}\left[\left(1-5 \cos ^{2} \theta\right) \widehat{\mathbf{r}}+2 \cos \theta \widehat{\mathbf{n}}\right] .
\end{aligned}
$$

The effects of the particles in motion mainly include the magnetic force, the viscous resistance of the magnetic particles and the carrier liquid due to the difference in speed during the movement, the repulsive force generated by the collision of the particles, and the repulsive force of the wall surface to prevent the particles from moving out of the wall surface.

2.2.1. Magnetic Force. The MRF microstructure simulated in this paper is under the action of a uniform magnetic field, and the magnetic field gradient is zero, so the magnetic particles are subjected to zero external force. It is assumed that all of the magnetic particles in the MRF have the same size and shape and are all circular. Then, the magnetic flux of the magnetic particles under the action of the magnetic field can be expressed as

$$
\mathbf{m}=\frac{4}{3} \pi R^{3} \chi \mathbf{H}
$$

In formula (23), $R$ is the radius of the magnetic particle, $\chi$ is MRF permeability; and $H$ is the magnetic field strength of the applied magnetic field.
According to the theory of magnetic dipole and Coulomb's law, the magnetic induction intensity produced by a magnetic dipole with a magnetic distance of $\mathbf{m}_{i}$ around it is as follows:

$$
\mathbf{B}=\frac{\mu_{0}}{4 \pi r^{3}}\left[\frac{3\left(\mathbf{m}_{i} \cdot \mathbf{r}\right) \mathbf{r}}{r^{2}}-\mathbf{m}_{i}\right]
$$

Then, in this magnetic field, the magnetic potential energy of another magnetic dipole with a magnetic moment of $\mathbf{m}_{j}$ is

$$
\mathbf{W}_{m_{i} m_{j}}=-\mathbf{m}_{j} \cdot \mathbf{B}=\frac{3 \mu_{0}}{4 \pi \mathbf{r}_{i j}^{3}}\left[\mathbf{m}_{i} \cdot \mathbf{m}_{j}-\frac{3\left(\mathbf{m}_{i} \cdot \mathbf{r}_{0}\right)\left(\mathbf{m}_{j} \cdot \mathbf{r}_{0}\right)}{r^{2}}\right] .
$$

In formula (25), $W$ is the relative position vector of any two magnetic particles and $Y$ is the unit vector.

It can be obtained that the magnetic force between the two magnetic particles is

$$
\mathbf{F}_{m_{i} m_{j}}=-\nabla \mathbf{W}_{m_{i} m_{j}}=\frac{3 \mu_{0}}{4 \pi \mathbf{r}_{i j}^{4}}\left[\mathbf{m}_{i} \cdot \mathbf{m}_{j}-3\left(\mathbf{m}_{i} \cdot \mathbf{r}_{0}\right)\left(\mathbf{m}_{j} \cdot \mathbf{r}_{0}\right)\right] .
$$

Therefore, the sum of the magnetic forces of any of the magnetic particles received by other magnetic particles is

$$
\mathbf{F}_{i}^{m}=\sum_{j \neq i} \frac{3 \mu_{0}}{4 \pi \mathbf{r}_{i j}^{4}}\left[\left(\mathbf{m}_{i} \cdot \mathbf{m}_{j}\right)-3\left(\mathbf{m}_{i} \cdot \mathbf{r}_{0}\right)\left(\mathbf{m}_{j} \cdot \mathbf{r}_{0}\right)\right] .
$$

2.2.2. Viscous Resistance. Because of the velocity difference between the magnetic particles and the carrier fluid in MRF, the particle motion is affected by resistance, so viscous resistance is produced. The viscous resistance of any magnetic particle $i$ in a stationary carrier fluid can be described by the Stokes formula as follows:

$$
F_{i}^{v}=-6 \pi R \eta \mathbf{v}_{i} .
$$

In formula (28), $R$ is the radius of the magnetic particles, $\eta$ is the MRF kinematic viscosity, and $\mathbf{v}_{i}$ is the velocity vector of the magnetic particles.

When the MRF shear deformation occurs under the action of the outside world, it is assumed that the MRF flows in the $x$ direction, and the applied magnetic field direction is perpendicular to the flow direction, that is, the $y$ direction. The shear strain rate is $\dot{\gamma}$. According to the relative motion principle, the viscous resistance of the magnetic particles can be reexpressed as

$$
F_{i}^{v}=-6 \pi R \eta\left(\mathbf{v}_{i}-y \dot{\gamma} \widehat{\mathbf{x}}\right) .
$$




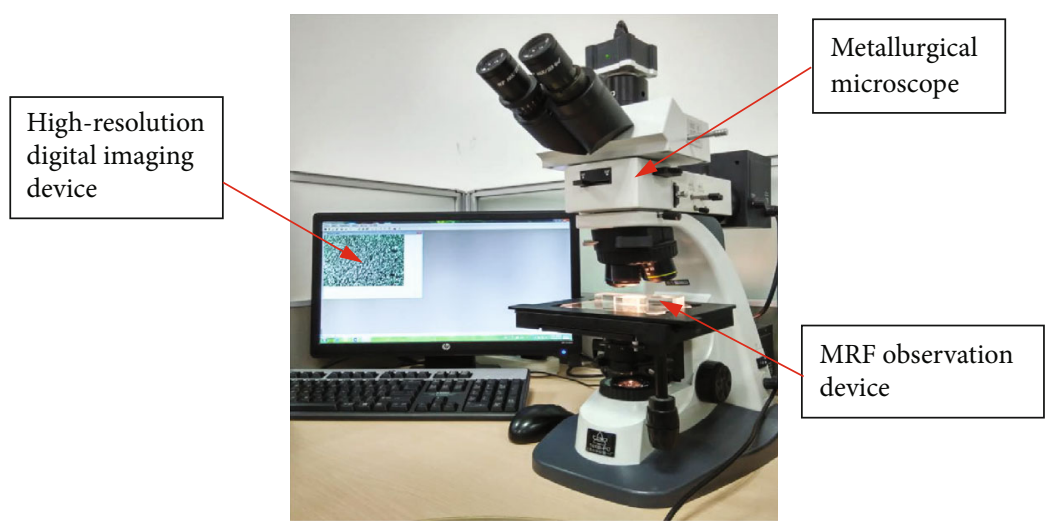

Figure 4: Static experimental device.

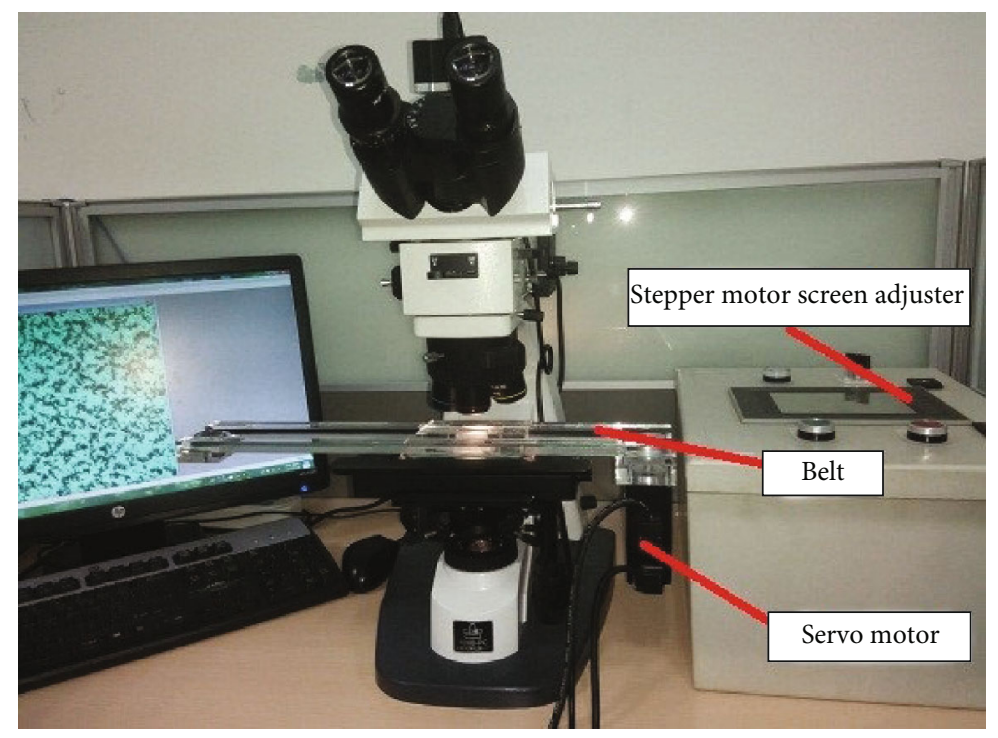

Figure 5: Experimental setup for shearing.

2.2.3. Repulsive Force. In MRF, there is a certain repulsive force between magnetic particles. Melle et al. [12] neglect the rotation of magnetic particles and do not consider the moment produced by the repulsive force. In an exponential form, the sum of the repulsive force of any magnetic particle $i$ by other particles around it is expressed as follows:

$$
F_{i}^{r}=\sum_{j \neq i} F_{0} \exp \left[-\beta\left(\frac{\mathbf{r}_{i j}}{2 R}-1\right)\right] \mathbf{r}_{i j} .
$$

In formula (30), $\beta$ is the material parameter, indicating that the repulsive force increases exponentially as the particle deformation increases, and $F_{0}$ is when any two magnetic particles are just in contact with the direction of the applied magnetic field; the magnetic force between the particles is maximum at this time. From the above analysis of the force of the magnetic particles, the resultant force of the magnetic particles is the sum of magnetic force, viscous resistance, and repulsive force. According to New- ton's second law, the equation of motion of magnetic particles can be expressed as follows:

$$
m a=m \frac{d^{2} r}{d t^{2}}=\sum F=F_{i}^{m}+F_{i}^{v}+F_{i}^{r}
$$

\subsection{Observation Experiment}

2.3.1. Static Observation Experiment. The MRF static observation device is shown in Figure 4. Two-dimensional observation of MRF is carried out by a metallographic microscope. The two-dimensional microstructures and twodimensional distribution of MRF in the absence of a magnetic field and in the presence of a magnetic field are obtained, respectively.

2.3.2. Dynamic Observation Experiment. The MRF shear observation experimental device is shown in Figure 5, and the working principle is as follows: the MRF is placed in the MRF channel formed by the upper and lower plates, and the baffle is placed on the rear side of the channel, and the 

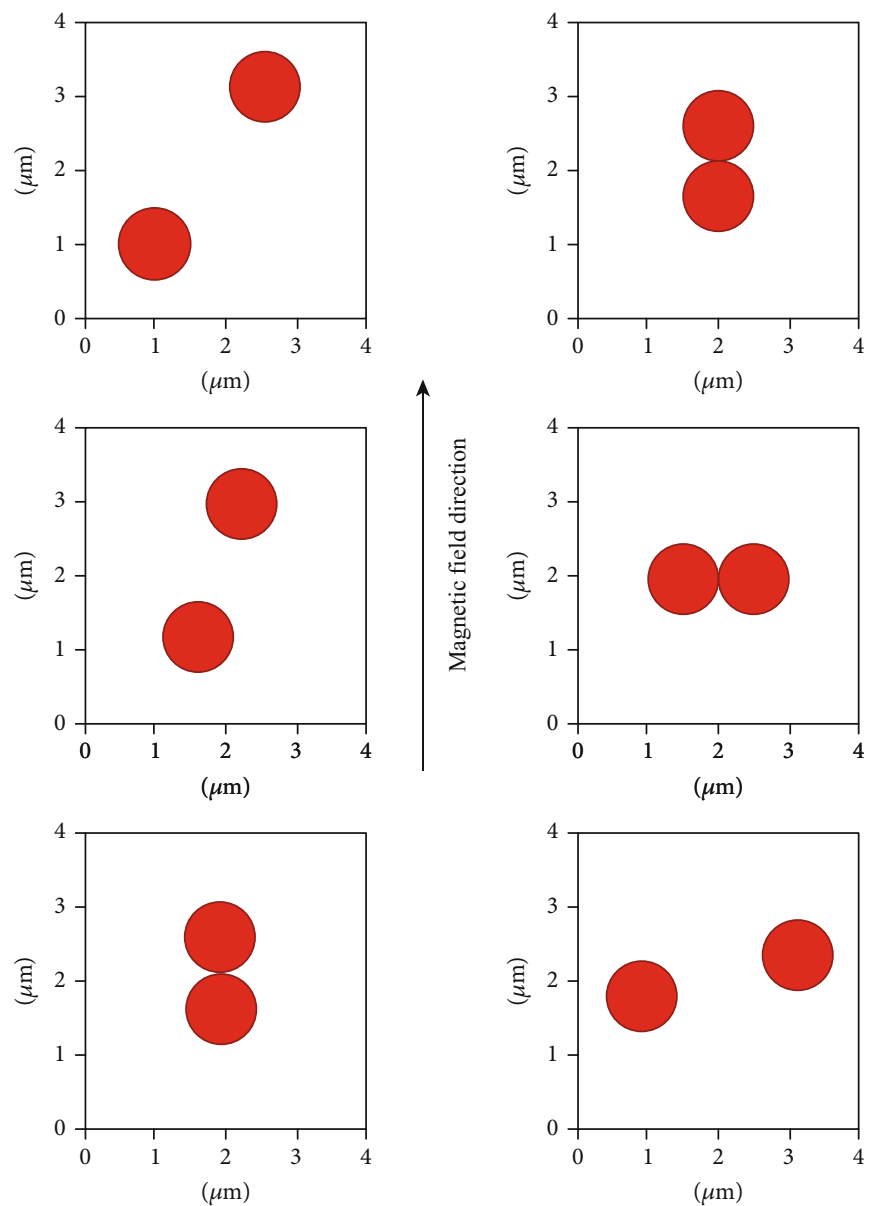

FIGURE 6: Simulation of the movement of two particles under a magnetic field.

baffle forms a shearing mechanism with the belt on the front side. A strong magnetic field is placed outside the baffle and belt to form a static magnetic field in the MRF channel. The magnetic induction intensity is measured by a Gaussian meter. The single-chip microcomputer sends a pulse to drive the stepping motor to drive the pulley to rotate, and the relative movement between the belt and the baffle causes shear deformation of the MRF in the channel. The macroshear strain rate can be controlled by the speed of the stepping motor.

In order to make the light transmittance good, the substrate is processed by a transparent acrylic plate, and the channel on which the MRF is placed on the observation plate is $50 \mathrm{~mm}$ long, $1.2 \mathrm{~mm}$ wide, and $5 \mathrm{~mm}$ deep. The two sides of the gap are belts, which are strong magnets on the outside of the two blocks. During the experiment, the MRF is placed near the center line of the strong magnetic height to make the applied magnetic field as uniform as possible.

\section{Comparison and Analysis of Numerical Simulation and Experimental Observation Results}

3.1. Analysis of Numerical Simulation Results. Firstly, by simulating the movement process of two magnetic particles
TABLE 1: The parameters used in simulation.

\begin{tabular}{|c|c|c|c|c|c|c|c|}
\hline $\begin{array}{l}L \\
(\mu \mathrm{m})\end{array}$ & $\begin{array}{c}W \\
(\mu \mathrm{m})\end{array}$ & $\begin{array}{c}R \\
(\mu \mathrm{m})\end{array}$ & $\begin{array}{c}\mu_{0} \\
(\mathrm{H} / \mathrm{m})\end{array}$ & $\begin{array}{c}\rho \\
\left(\mathrm{kg} / \mathrm{m}^{3}\right)\end{array}$ & $\begin{array}{c}\eta \\
(\mathrm{pa} \cdot \mathrm{s})\end{array}$ & $\begin{array}{c}r_{\mathrm{c}} \\
(\mu \mathrm{m})\end{array}$ & $\chi$ \\
\hline 400 & 400 & 5 & $4 \pi \times 10^{-7}$ & $7.5 \times 10^{3}$ & 0.001 & 30 & 1. \\
\hline
\end{tabular}

under the action of a magnetic field, the process of MRF chaining is initially understood. Its movement is divided into two situations: One is that the angle between the central line of two magnetic particles and the direction of external magnetic field is relatively small. The other is that the central line of two magnetic particles is nearly perpendicular to the angle of magnetic field direction. The specific simulation process is shown in Figure 6, where the left column is the motion simulation of the former case and the right column is the motion simulation of the latter case. It can be seen that the movements of the two are very different. The former attracts each other and closes, and gradually aligns along the direction of the magnetic field; the latter repel each other, and the particles are further away.

3.1.1. Influence of Magnetic Induction. After defining the movement trend of two magnetic particles under the action of a magnetic field, in order to describe the actual situation 


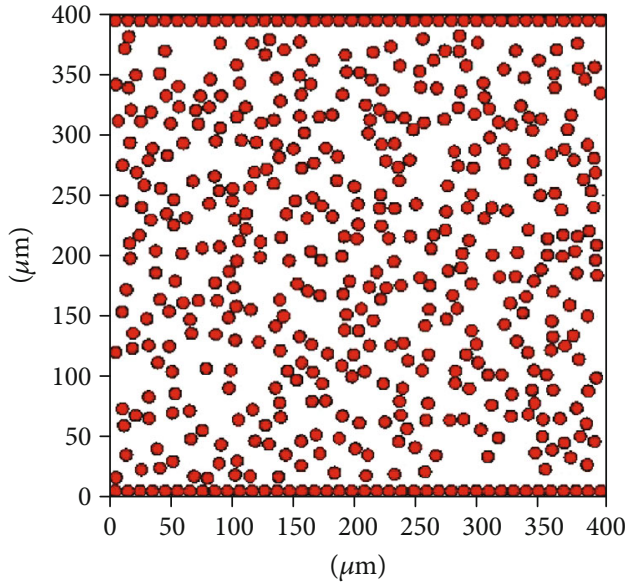

(a) $B=0 \mathrm{Gs}$

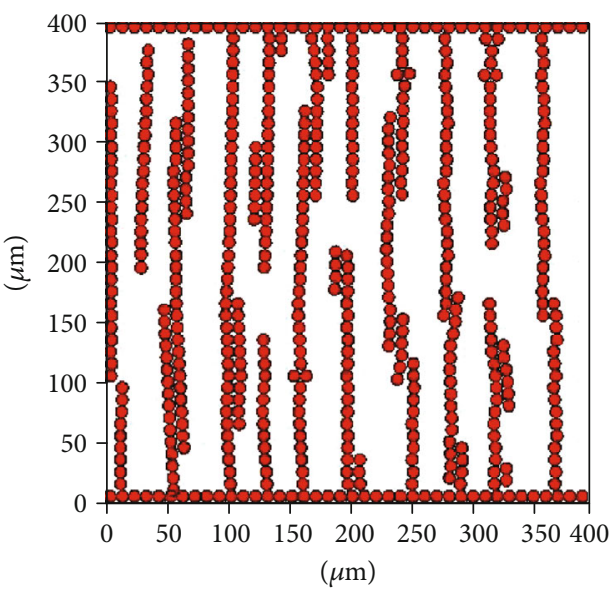

(c) $B=100 \mathrm{Gs}$

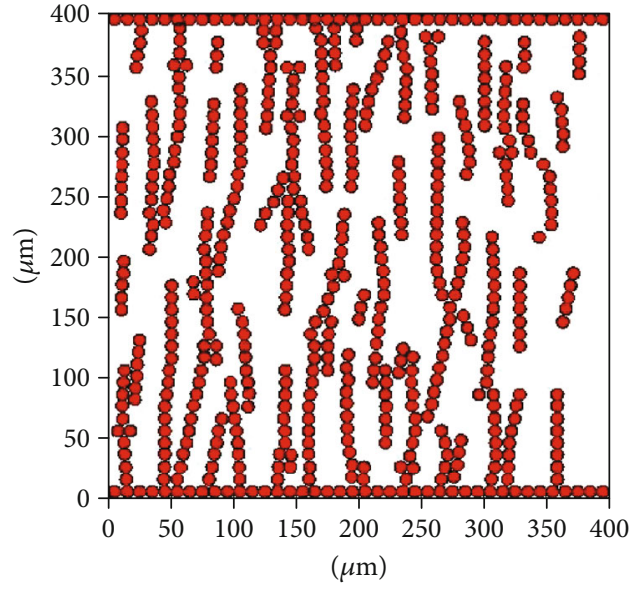

(b) $B=50 \mathrm{Gs}$

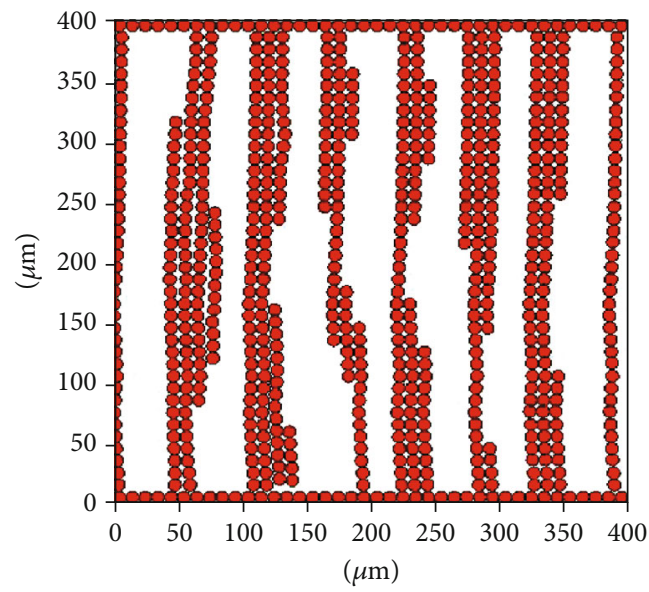

(d) $B=250 \mathrm{Gs}$

FIgURE 7: Chain simulation results of MRF.

of the MRF chaining process in more detail, a twodimensional simulation region was taken to simulate the process of forming a chain structure along the direction of the magnetic field for several magnetic particles under the action of an external magnetic field with different magnetic induction intensities. The magnetic induction intensity $\mathrm{B}$ was $0 \mathrm{Gs}, 50 \mathrm{Gs}, 100 \mathrm{Gs}$, and $250 \mathrm{Gs}$ in turn. The basic simulation parameters are shown in Table 1, where the direction of the magnetic field is vertically downward.

Among the parameters are $L$ and $W$, which represent the length and width of the simulated two-dimensional region, respectively; $R$, which represents the radius of magnetic particles; $\mu_{0}$, which denotes vacuum permeability; $\rho$, which represents the magnetic particle density; $\eta$, which represents the viscosity coefficient of the carrier liquid; $r_{c}$, which represents the cutoff radius; and $\chi$, which indicates the magnetic susceptibility.

After defining and setting the basic parameters of magnetic particles in the program, the simulation results of the MRF chain process are obtained by running the program as shown in Figure 7. As can be seen from the figure, at $B=0$ Gs, magnetic particles are randomly distributed in the twodimensional simulation area. As shown in Figure 7(a), once an external magnetic field is applied, the magnetic particles gather together within a short time under the action of a magnetic field to form a plurality of short particle chains and clusters of particles [13]. As shown in Figure 7(b), with the increase of magnetic induction intensity, these short chains and clusters will gather together, which will increase the length of the particle chain, thus forming a through chain and forming a bundle chain structure. As shown in Figure $7(\mathrm{c})$, when the magnetic induction intensity reaches $250 \mathrm{Gs}$, several through chains gather together to form multirow chains. And finally, the chain-like microstructures along the magnetic field direction are obtained. It can be seen that when the microstructure of the MRF reaches a steady state, most of the particles exist in the form of a chain, but there are branches, isolated chains, and bundles at the same time, and not all the particles are strictly along the magnetic field. Arranged in one direction, a part of the chain is tilted due to the influence of other particles and adjacent chains.

3.1.2. Influence of Particle Size. Considering the influence of the size of magnetic particles on the chaining process of the microstructure of MRF, four different radii of magnetic 


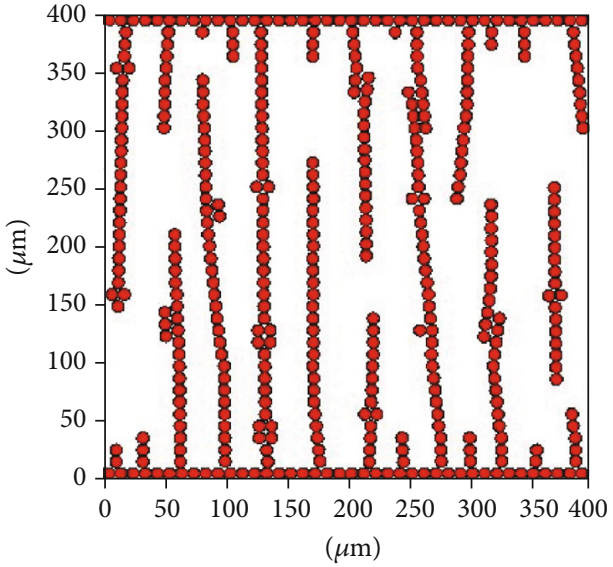

(a) $R=0.5 \mu \mathrm{m}$

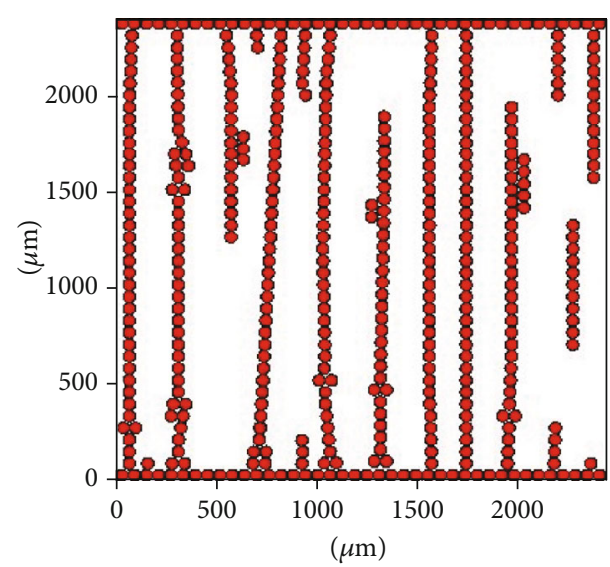

(c) $R=30 \mu \mathrm{m}$

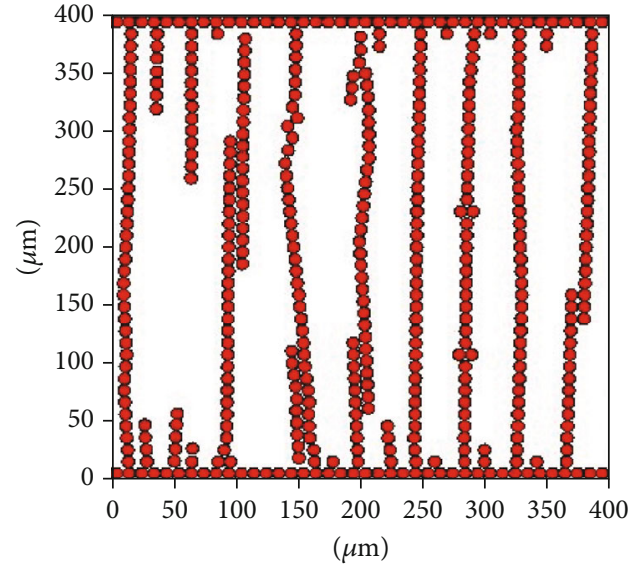

(b) $R=5 \mu \mathrm{m}$

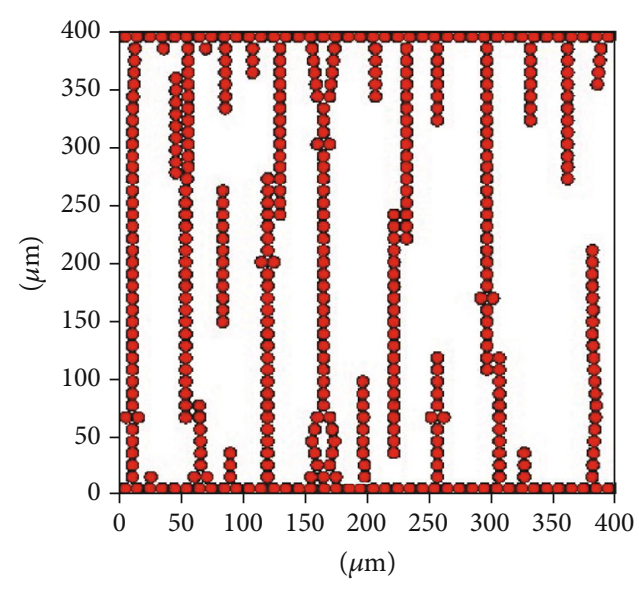

(d) $R=50 \mu \mathrm{m}$

FiguRE 8: Microstructures of different particle sizes are simulated.

particles were used in the simulation. The magnetic structure of the MRF was simulated by magnetic particles with radiuses of $R=0.5 \mu \mathrm{m}, 5 \mu \mathrm{m}, 30 \mu \mathrm{m}$, and $50 \mu \mathrm{m}$, respectively [14]. The simulation results are shown in Figure 8. It can be seen from Figure 8 that although the particle size has a large difference, the microstructure of the MRF obtained by the simulation is substantially similar, so the particle size has little effect on the microchain structure of the MRF.

3.1.3. Influence of Particle Volume Fraction. The volume fraction of the particles has a great influence on the performance of MRF. In order to study the effect of particle volume fraction on the MRF chain structure, the chain structure of MRF at $\varphi=5 \%, 10 \%$, and $30 \%$ is simulated, respectively. At the same time, in order to further verify the influence of particle size on the chain structure, two different radii particles were used in the simulation, respectively, $R=5 \mu \mathrm{m}$ and $R=50 \mu \mathrm{m}$. The simulation results are shown in Figure 9.

It can be seen from the figure that when the volume fraction of particles is very small, the particles mostly exist in the form of isolated chains and branched chains. At this time, the chain length is relatively short and there are still some single particles. With the increase of volume fraction, there are straight chains in the simulation area. The length of isolated chains and branched chains increases and the number of isolated chains decreases. There are almost no single particles. With the increase of volume fraction, the number of through chains increases. At the same time, the isolated chains disappear and some particle chains aggregate to form bundle chains. When the particle volume fraction is large, most of the particle chains exist in the form of bundle chains.

At the same time, in order to better study the microstructure of MRF under shear force and the effect of different shear strain rates on the microstructure of MRF, the MRF with a particle radius of $R=5 \mu \mathrm{m}$ and a volume fraction of $\varphi=10 \%$ was simulated. The process of microstructure chaining was simulated during the shear strain rates of $100 \mathrm{~s}^{-1}$ and $800 \mathrm{~s}^{-1}$. It can be concluded that the direction of the particle chain along the shearing motion during the shearing process is inclined in a direction perpendicular to the magnetic field, thereby forming a certain angle with the direction of the magnetic field; as the shearing motion continues, the angle increases. When it becomes large and increases to a certain value, the particle chain will break, and at the same 


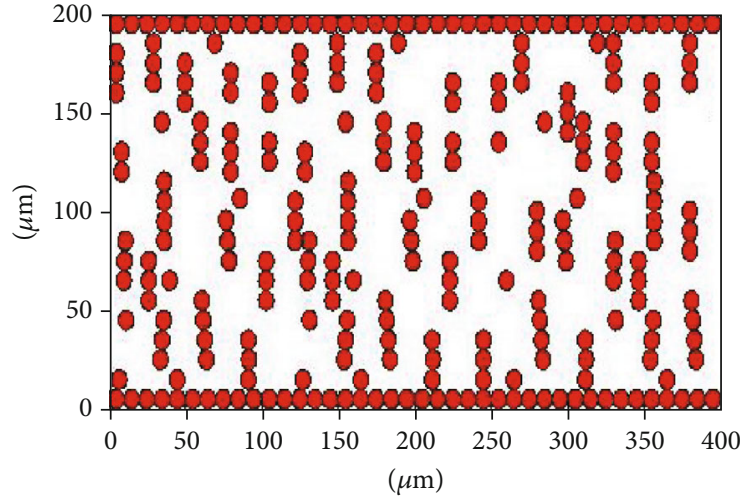

(a) $\varphi=5 \%(R=5 \mu \mathrm{m})$

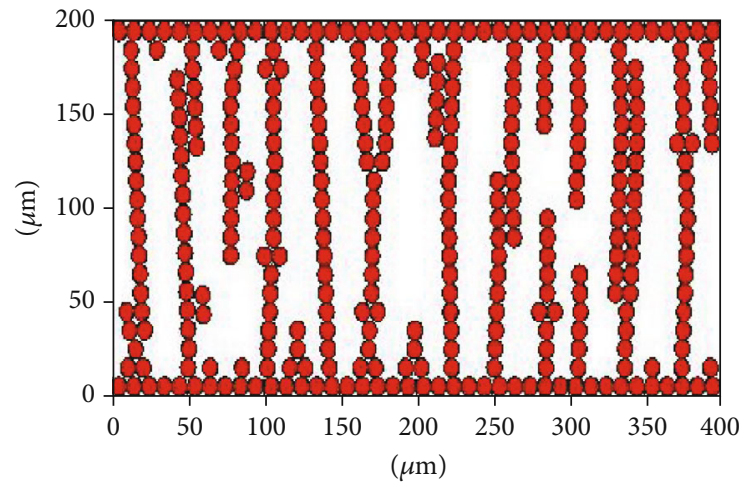

(c) $\varphi=10 \%(R=5 \mu \mathrm{m})$

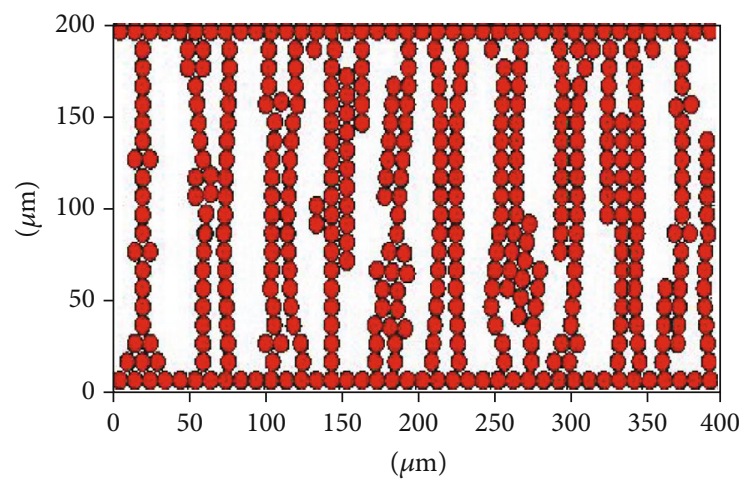

(e) $\varphi=30 \%(R=5 \mu \mathrm{m})$

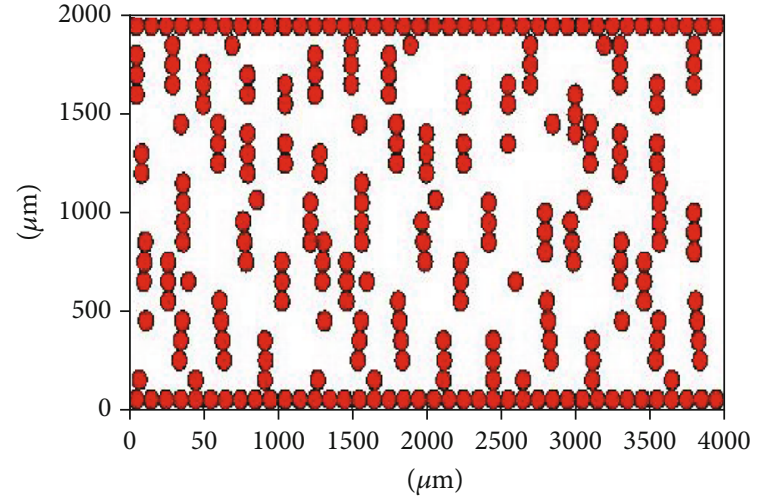

(b) $\varphi=5 \%(R=50 \mu \mathrm{m})$

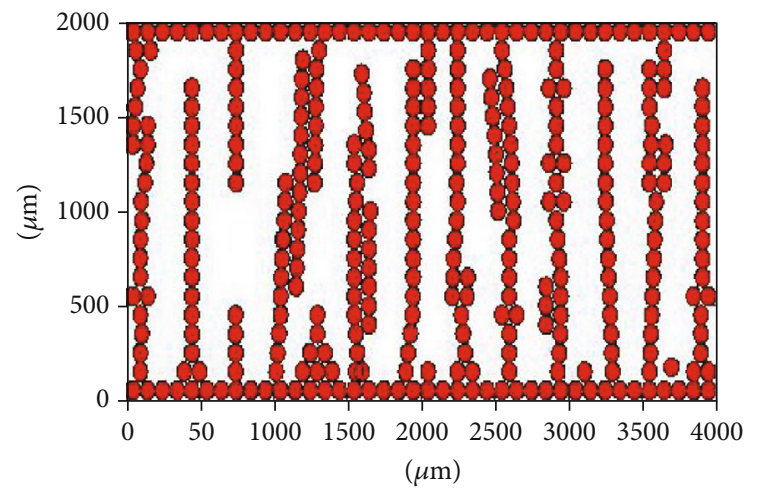

(d) $\varphi=10 \%(R=50 \mu \mathrm{m})$

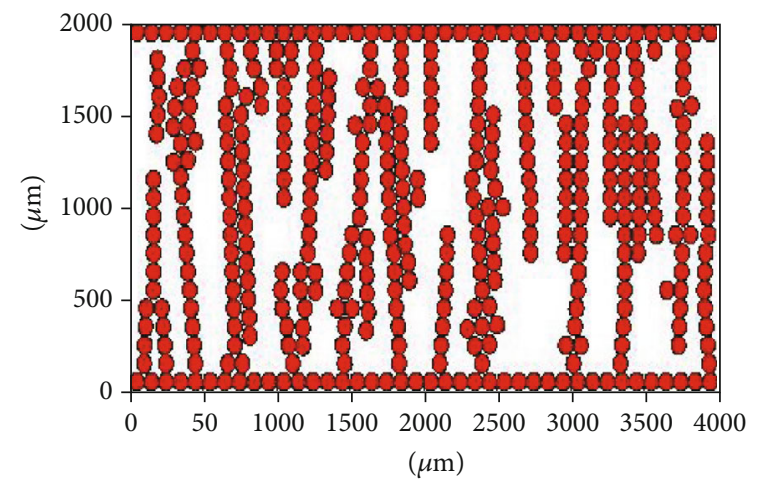

(f) $\varphi=30 \%(R=50 \mu \mathrm{m})$

Figure 9: Microstructures of different particle sizes are simulated.

time some new particle chains will form [15]. It is not that the particle chains used break at the same time, but that at a certain time, a part of the particle chains break under the action of shear force, and at the same time, a part of the new particle chains will be generated. This can well explain the macroscopic phenomenon that the shear stress of MRF decreases with the increase of shear strain rate when the shear strain rate is large.

\subsection{Analysis of Experimental Observations}

\subsubsection{Static Observation Experiment Results}

(1) Influence of the Nonmagnetic Field. The black parts are the magnetic particles, and the white part is the liquid

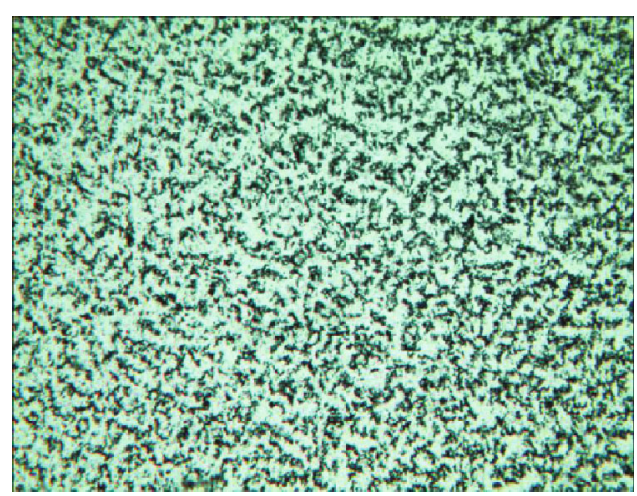

FIgURE 10: Microstructure of magnetorheological fluids without a magnetic field. 


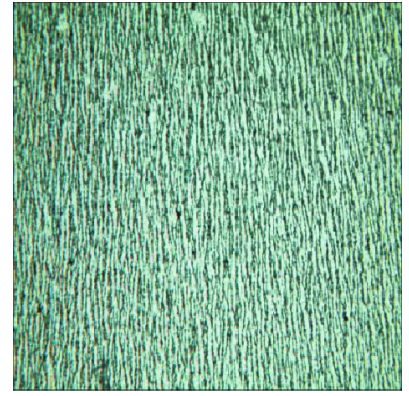

(a) $B=50 \mathrm{Gs}$

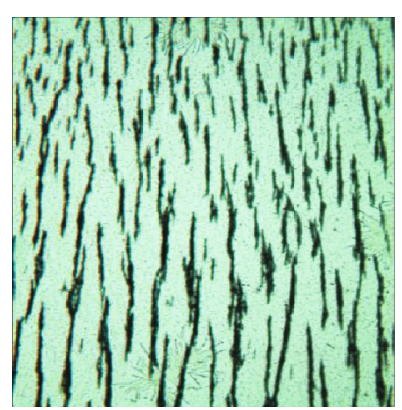

(b) $B=150 \mathrm{Gs}$

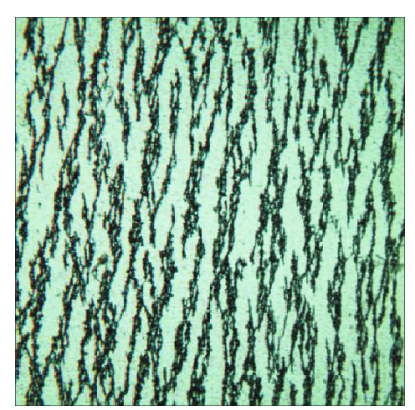

(c) $B=250 \mathrm{Gs}$

FIGURE 11: MRF microstructure under different magnetic induction strengths.

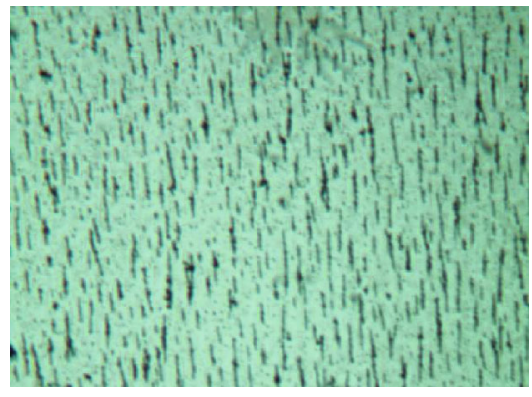

(a) $\varphi=5 \%$

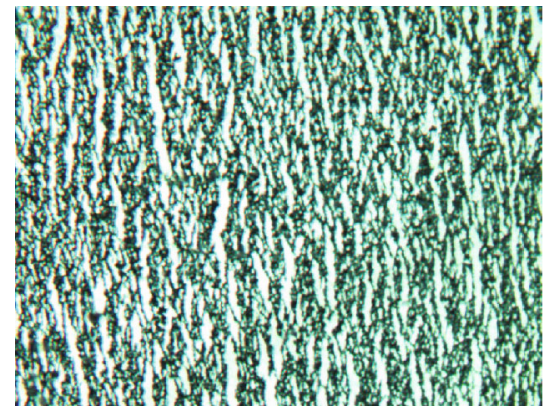

(b) $\varphi=30 \%$

FIGURE 12: MRF microstructures with different particle volume fractions.

carrier. The microstructure was observed with a 50 -fold magnification objective, and the magnification of the image was 500 -fold on a computer by a digital imaging device.

The MRF used in this experiment was prepared by using carbonyl iron powder as magnetic particles and dimethyl silicone oil as base liquid, with the addition of a certain amount of additives [16]. In the observation experiment, three volume fractions of MRF were used, and the volume percentages of carbonyl iron powder were $5 \%, 15 \%$, and $30 \%$, respectively. The MRF sample with a volume fraction of $15 \%$ was selected as the observation solution. The observation results are shown in Figure 10. It can be seen from the figure that for MRF composed of micron-sized magnetic particles, magnetic particles and their particle clusters can be clearly observed at a magnification of 500 times, and magnetic particles are substantially uniformly distributed for MRF having a volume fraction of $15 \%$. At the first level, the resulting image is also relatively clear. When no external magnetic field is applied, the magnetic particles are randomly distributed in the carrier liquid, and it can be found that it has good fluidity.

(2) Influence of Magnetic Induction Intensity. In order to observe the influence of magnetic field strength on the microstructure of MRF, in the experiment, MRF with a volume fraction of $15 \%$ was also selected as the sample to be observed. By applying magnetic fields of different strengths, the microstructure of MRF as showed in Figure 11 can be obtained. By comparison, it can be seen that with the increase of the magnetic induction intensity [17], the microchain structure of a magnetic chain is also different. In Figure 11(a), the chain structure is more uniform and the slender chain is the main one. With the increase of the magnetic field, as shown in Figure 11(b), the chain becomes thicker and the interaction between the chain and the chain structure becomes more obvious. From the same field of view, Figure 11(c) shows that the chain structure is obviously coarsened, and the chain and chain structure combine to form a more obvious chain column structure. In summary, with the change of magnetic field, the microstructures formed by ferromagnetic particles also change: from the initial irregular distribution to the regular chain structure, and then some magnetic chains gradually combine to form chain columns. It can be seen that the magnitude of the applied magnetic field is one of the important factors affecting the morphology of MRF microstructures.

(3) Effect of Particle Volume Fraction. In order to study the effect of particle volume fraction $\varphi$ on the MRF microstructure chaining process [18], the microscopic chain structure of MRF samples with magnetic particle volume fractions of $5 \%$ and $30 \%$ of magnetic induction $B=250 \mathrm{Gs}$ was observed experimentally, as Figure 12 shows.

It can be seen from Figure 12 that when the particle volume fraction is small, the microstructure of MRF is 


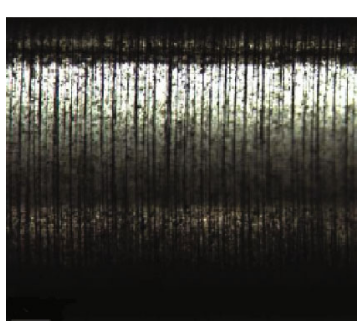

(a) $t=0 \mathrm{~s}$

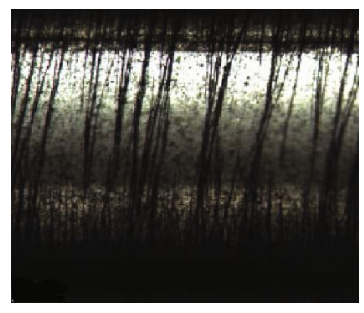

(d) $t=3 \mathrm{~s}$

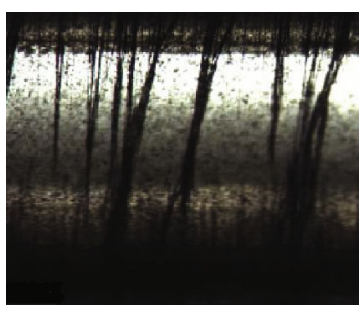

(g) $t=6 \mathrm{~s}$

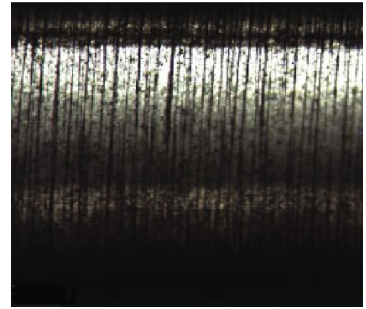

(b) $t=1 \mathrm{~s}$

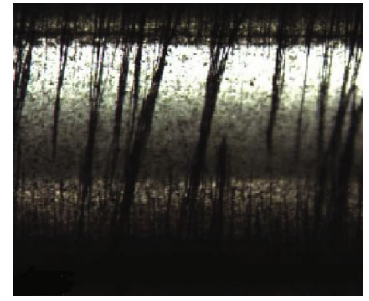

(e) $t=4 \mathrm{~s}$

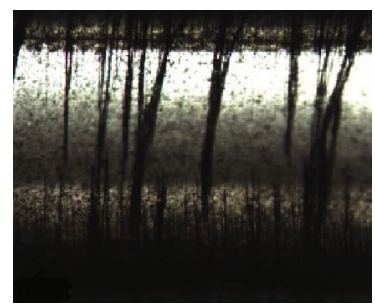

(h) $t=7 \mathrm{~s}$

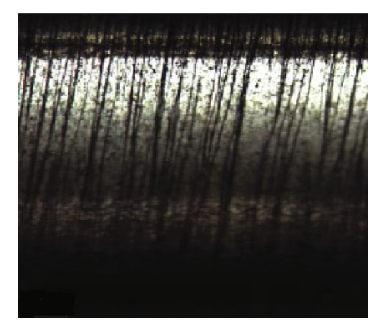

(c) $t=2 \mathrm{~s}$

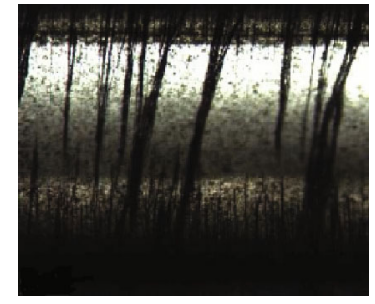

(f) $t=5 \mathrm{~s}$

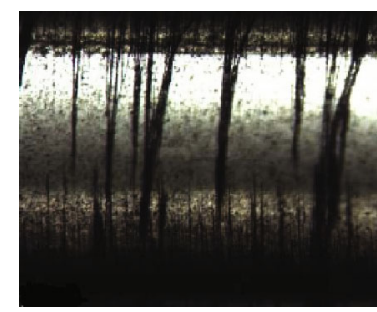

(i) $t=8 \mathrm{~s}$

Figure 13: Dynamic evolution of magnetorheological fluid microstructure.

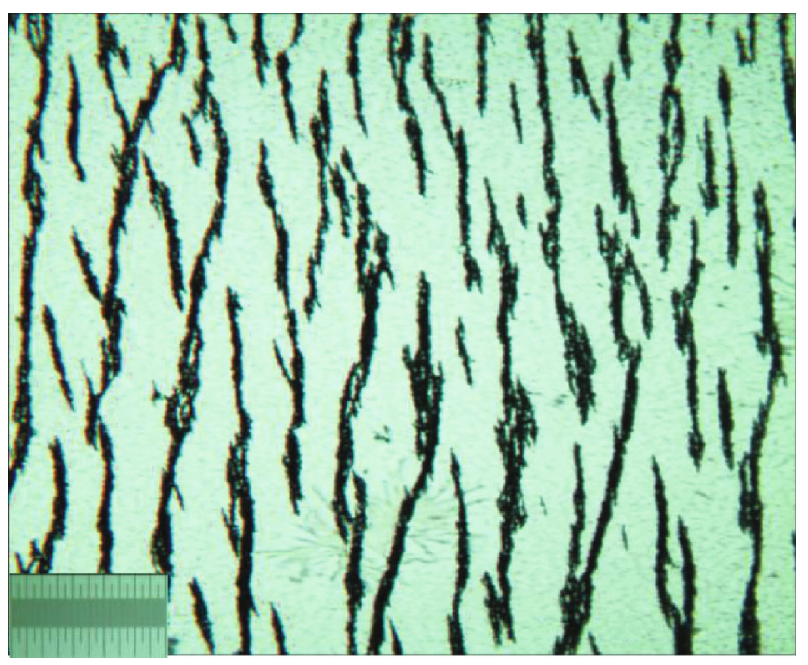

FIGURE 14: Chain spacing measurement chart.

independent of each other. There is no through chains and branching chains in the field of view. Particles exist in the form of branched chains, isolated chains, and free particles. At this time, the gap between particle chains is larger. With the increase of the particle volume fraction, the number of through-chains also increases. When the particle volume fraction increases to $\varphi=30 \%$, the particle chains mostly exist in the form of bundle chains. The cross-links between the particle chains form a column chain. It can be clearly seen that the microchain structure

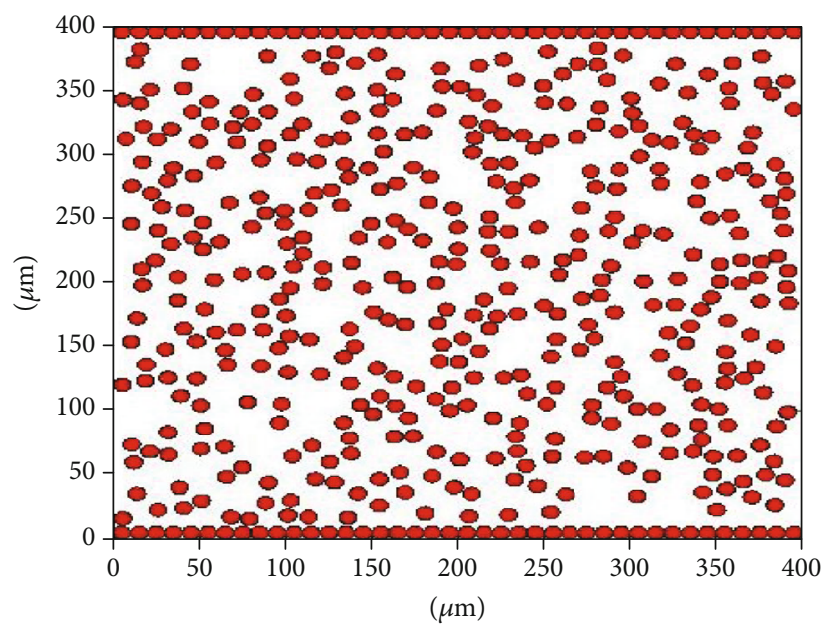

FIgURE 15: Magnetic particle simulation map without a magnetic field.

is relatively dense, and the gap between the column chains is small.

3.2.2. Experimental Results of Dynamic Observation. During the test, the sample was evenly stirred, and a little was taken out in the observation tank. The shooting was performed in an automatic capture mode with a time interval of $1 \mathrm{~s}$, an imaging magnification of 500 times, and a moving speed of about $1 \mathrm{~mm} / \mathrm{s}$. The shooting results are shown in Figure 13. When $t=0 \mathrm{~s}$, the MRF forms a chain 


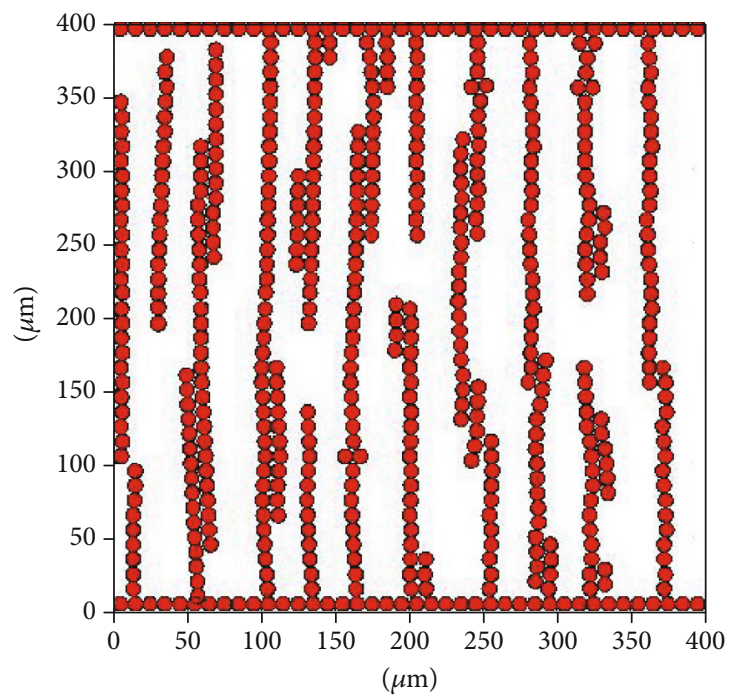

(a) $B=100$ Gs magnetic particle simulation distribution map

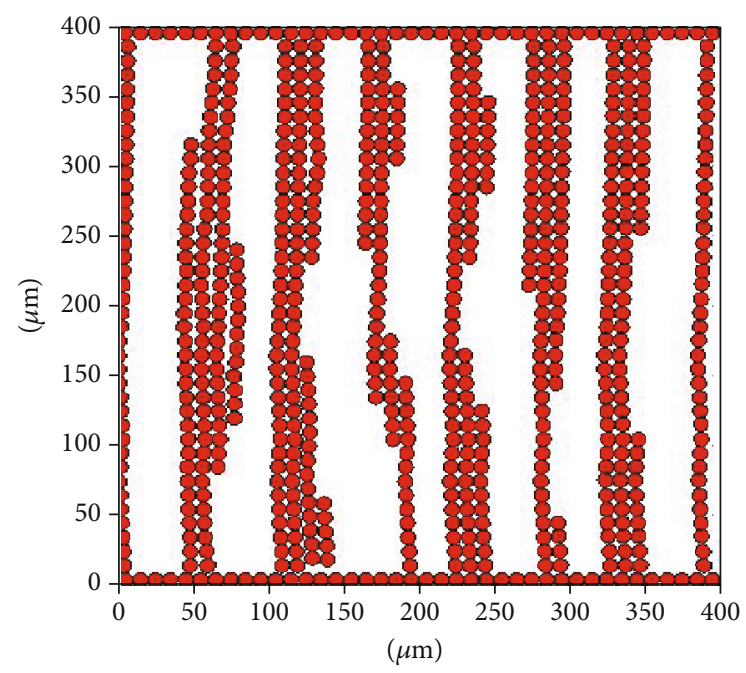

(c) $B=250$ Gs magnetic particle simulation distribution map

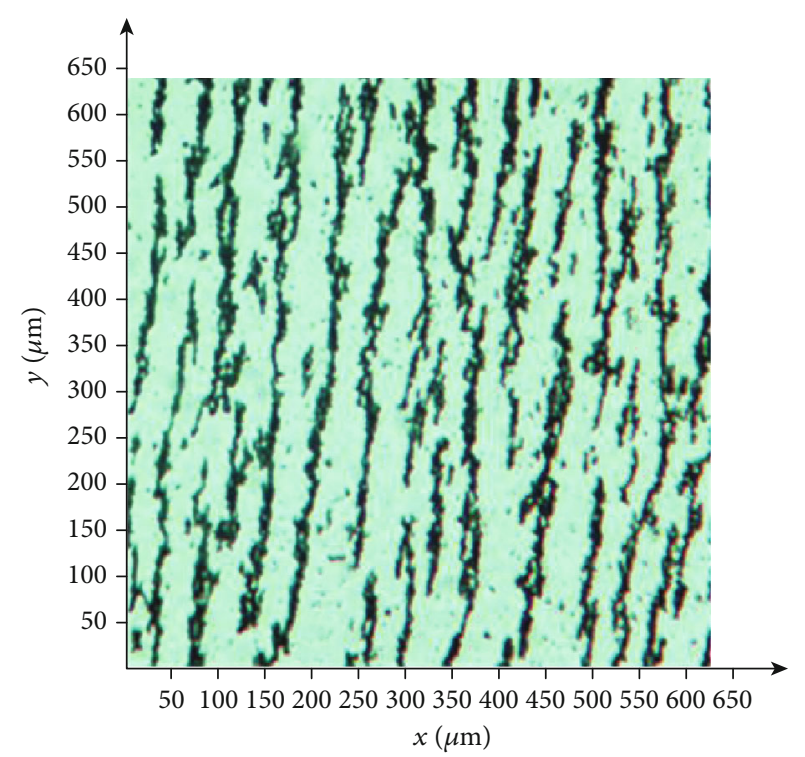

(b) $B=100$ Gs magnetic particle test distribution map

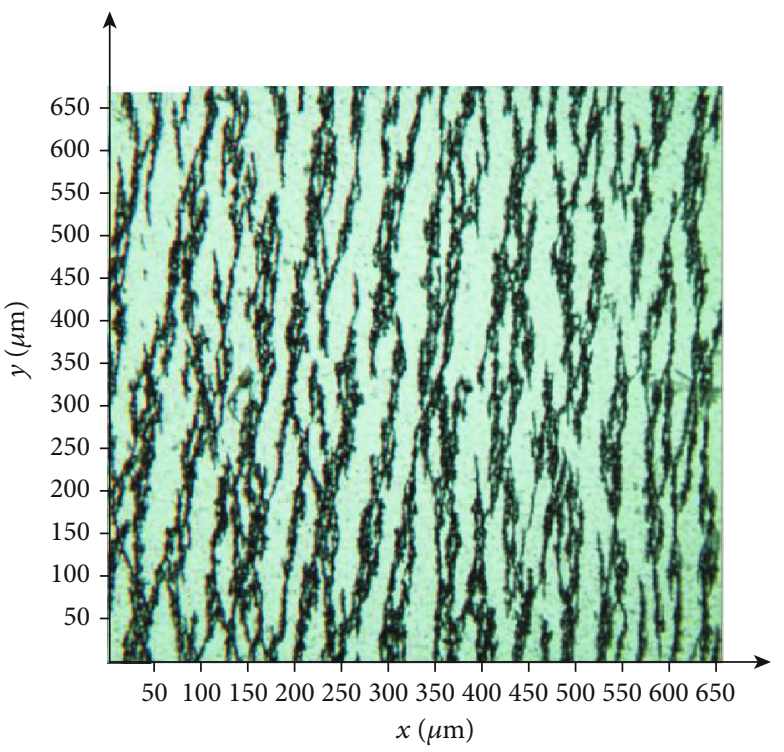

(d) $B=250$ Gs magnetic particle test distribution map

FIgURE 16: Comparison of different magnetic induction intensities.

structure which is stable along the direction of the magnetic field under the action of an external magnetic field, and the thickness and distribution of the chain in the field of view are substantially uniform. When $t=1 \mathrm{~s}$, as the lower base plate tilts to the left kinematic chain, the shape and density of the chain do not change significantly due to the smaller dip angle. When $t=2 \mathrm{~s}$, as the belt moves further to the left, some adjacent chains merge to form a thicker chain during the tilting process, and the density of the chain or chain is reduced. In the right end of the figure, it can be found. The individual chains are disconnected from the middle portion, a part of which continues to move with the belt, and the other part remains in the carrier liquid in the form of a broken chain and is kept inclined under the action of the magnetic force and the carrier liquid, and has some bending deformation. At $t=3 \mathrm{~s}$,
TABle 2: Average distance between particle chains under different magnetic induction.

\begin{tabular}{lcc}
\hline & \multicolumn{2}{c}{$\begin{array}{c}\text { Average distance between } \\
\text { particle chains }(\mu \mathrm{m})\end{array}$} \\
& $B=100 \mathrm{Gs}$ & $B=250 \mathrm{Gs}$ \\
\hline Analog value & 34.23 & 30.85 \\
Experimental value & 36.53 & 32.56 \\
Relative error (\%) & $6.3 \%$ & $5.3 \%$ \\
\hline
\end{tabular}

with the further combination of adjacent chains to form thicker chains, the density of chains or chains decreases further, the inclination angle increases, and some chains appear slightly fan-shaped. When $t=4-5 \mathrm{~s}$, the chain or chain column appears obviously fan-shaped. Some chains or chain 


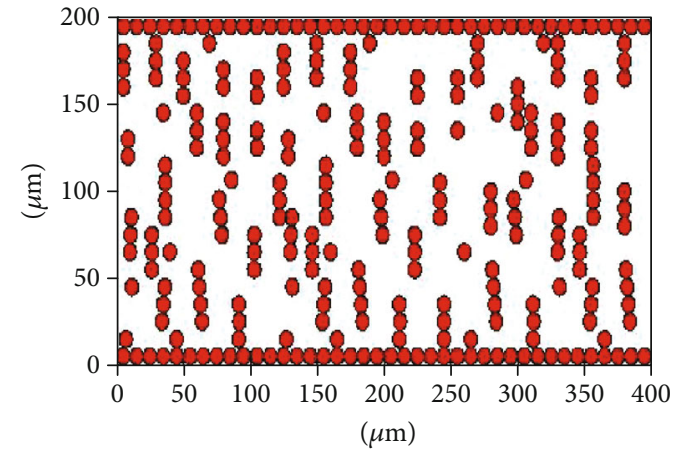

(a) $5 \%$ magnetic particle simulation map

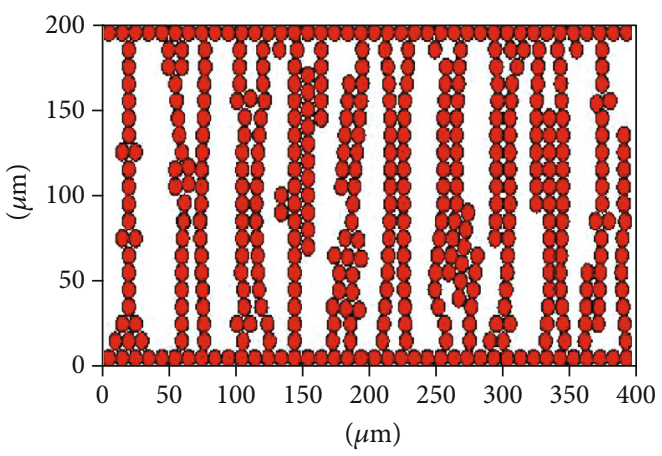

(c) 30\% magnetic particle simulation map

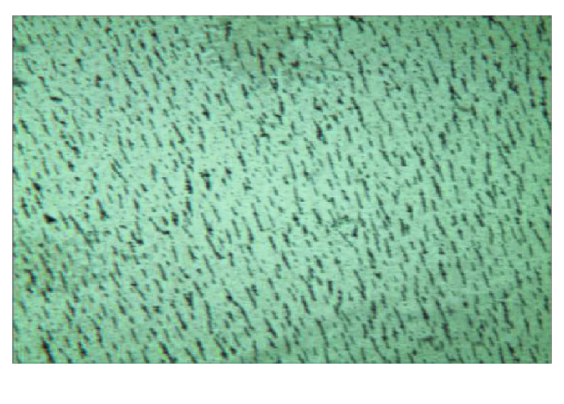

(b) $5 \%$ magnetic particle test distribution

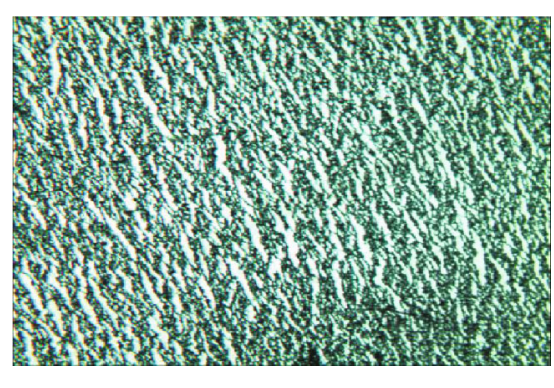

(d) 30\% magnetic particle test distribution

FIGURE 17: Comparison chart of different particle volume fractions.

TABle 3: Average distance between particle chains under particle volume fraction.

\begin{tabular}{lcc}
\hline & \multicolumn{2}{c}{$\begin{array}{c}\text { Average distance between } \\
\text { particle chains }(\mu \mathrm{m})\end{array}$} \\
& $5 \%$ & $30 \%$ \\
\hline Analog value & 35.52 & 28.14 \\
Experimental value & 37.26 & 29.37 \\
Relative error $(\%)$ & $4.7 \%$ & $4.2 \%$ \\
\hline
\end{tabular}

columns are pulled off from the middle. The broken chain tends to the direction of the magnetic field and merges into the left chain with the movement of the plate to form a new thick chain column. When $t=6-8 \mathrm{~s}$, the density of the chain in the carrier fluid basically does not change at this time, and the shape of the chain is mainly the thicker broken chain column and through chain column, which can be attributed to the failure of the test device to provide periodic or continuous shear deformation.

3.3. Analysis of Comparative Results. In this paper, the magnetorheological fluid chain spacing is measured by taking microscopic observation pictures and the scale of the $\mathrm{C} 1$ type micrometer $(1 / 100)$ at the same magnification. The microscopic observation map and the micrometer scale of the stage are the same pixels and the same scale. Therefore, the microscopic picture can be measured with the scale of the shooting.
The specific measurement chart is shown in Figure 14. The corresponding distance value can be read by the scale on the graph.

3.3.1. Influence of the Nonmagnetic Field. When there is a nonmagnetic field, the magnetic particles are randomly distributed. The simulation figure is shown in Figure 15. It can be seen that the particles are randomly distributed in the carrier fluid.

3.3.2. Influence of Magnetic Induction Intensity. Once applied, the magnetic field rapidly forms a chain along the direction of the magnetic field. Most of the particles exist in the form of through-chains [19], but some chains are inclined due to the influence of other particles and adjacent chains. At the same time, with the increase of magnetic induction intensity, the interaction force between particle chains increases, particle chains aggregate, the average length of chains increases, the number of isolated chains decreases, the number of bundles increases, and the distance between chains increases until the isolated chains disappear completely, and particle chains cross-link due to mutual attraction. The comparison between numerical simulation and experimental results is shown in Figure 16.

The measurement results of the numerical simulation map are compared with the measurement results of the experimental photograph, as shown in Table 2.

From the above table, the relative errors of the average distances between the simulated and experimental particle chains are $6.3 \%$ and $5.3 \%$, respectively, under the conditions of $B=100 \mathrm{Gs}$ and $B=250 \mathrm{Gs}$. At the same 


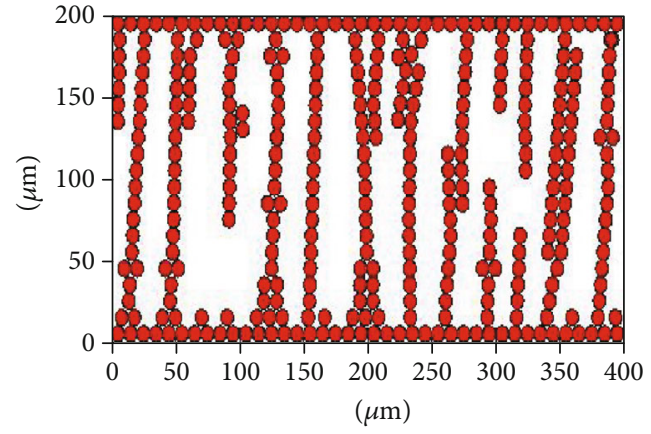

(a) Shear strain rate $=100 \mathrm{~s}^{-1}$ magnetic particle simulation map

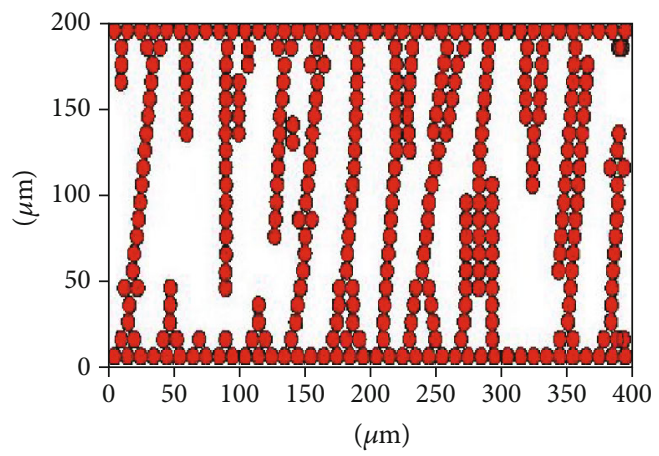

(c) Shear strain rate $=800 \mathrm{~s}^{-1}$ magnetic particle simulation map

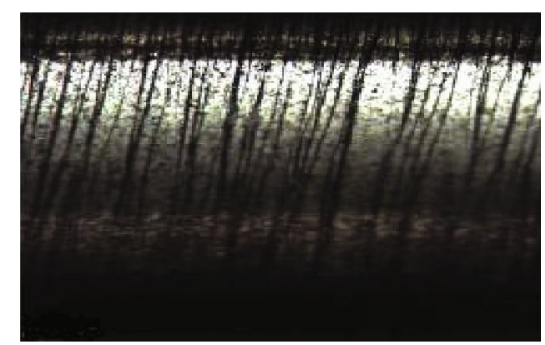

(b) Shear strain rate $=100 \mathrm{~s}^{-1}$ magnetic particle test distribution

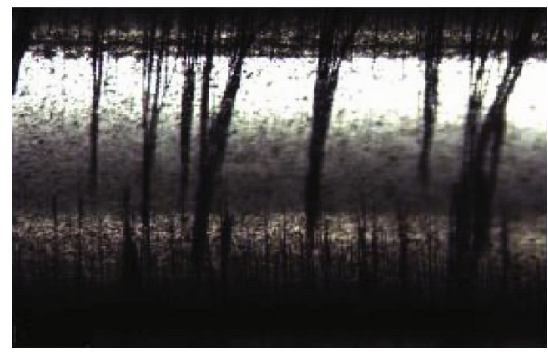

(d) Shear strain rate $=800 \mathrm{~s}^{-1}$ magnetic particle test distribution

Figure 18: Comparison of shear force.

TABLE 4: Angle between the particle chain and the direction of the magnetic field at different shear strain rates.

\begin{tabular}{lcr}
\hline & $\begin{array}{c}\text { The angle between the particle chain and the direction of the magnetic field }\left(^{\circ}\right) \\
\text { Shear strain rate }=100 \mathrm{~s}^{-1}\end{array}$ \\
Shear strain rate $=800 \mathrm{~s}^{-1}$
\end{tabular}

time, it can be seen that the average distance between particle chains decreases with the increase of magnetic field intensity.

3.3.3. Influence of Particle Volume Fraction. When the particle volume fraction $\varphi$ is small, the microstructure of the MRF is independent of each other, and there are no through chains and branches in the field of view. The particles exist in the form of branches, isolated chains, and free particles. The gap between the chains is large. When the particle volume fraction $\varphi$ is large [20], the particle chains are mostly in the form of bundle chains, and the particle chains are crosslinked together to form a column chain. It can be clearly seen that the microchain structure is dense, and the gap between each column chain is small. A comparison of the numerical simulation and experimental results is shown in Figure 17.

Table 3 shows that the relative errors of the average distances between the simulated and experimental particle chains are $4.7 \%$ and $4.2 \%$ under the conditions of $5 \%$ and $30 \%$ of the particle volume fraction, respectively. At the same time, it can be seen that the average distance between particle chains decreases with the increase of the percentage of particle volume under the action of constant magnetic field.

3.3.4. Influence of Shear Strain Rate. During the shearing process, the particle chains are inclined in the direction perpendicular to the magnetic field in the direction of the shearing motion, thereby forming a certain angle with the direction of the magnetic field. The shear strain rate is different [21], and the angle formed by it is also different. A comparison of the numerical simulation and experimental results is shown in Figure 18.

The measurement results of the numerical simulation map are compared with the measurement results of the experimental photographs, as shown in Table 4.

According to the above table, the relative errors of the angle between the particle chain and the magnetic field are $4.0 \%$ and $3.1 \%$ under the conditions of $100 \mathrm{~s}^{-1}$ and $800^{s-1}$ shear strain rates, respectively. At the same time, it can be seen that under the action of a constant magnetic field, the angle between the particle chain and the magnetic field decreases with the increase of the applied shear strain rate. 


\section{Conclusions}

In this paper, the simulated and experimental values of MRF microstructure chaining process were compared in the four aspects of MRF microstructure chaining process, including magnetic field strength, magnetic induction intensity, particle volume fraction, and shear strain rate. The following results are compared:

(1) Microstructure comparison of MRF under a nonmagnetic field and a magnetic field

(2) The average distance between the particle chains of the numerical simulation and experimental results under the conditions of $B=100 \mathrm{Gs}$ and $B=250 \mathrm{Gs}$

(3) The average distance between the particle chains of numerical simulation and experimental results under the conditions of particle volume percentages of $5 \%$ and $30 \%$, respectively

(4) The angle between the particle chain and the magnetic field in the numerical simulation and experimental results under the conditions of shear strain rate of $100 \mathrm{~s}^{-1}$ and $800 \mathrm{~s}^{-1}$, respectively.

According to the comparison experiment and numerical simulation results, under the action of constant magnetic field, the angle between the particle chain and the magnetic field decreases with the increase of the applied shear force. The analysis results show that the maximum relative error is less than $7 \%$, which indicates that the simulation model established in this paper has high reliability. When the shear strain rate is $800 \mathrm{~s}^{-1}$, the relative error is $3.1 \%$, and the simulation result is ideal. While enriching the microchained structure of MRF, it also has a certain significance for the improvement of the MRF preparation formula and design, research, and development of automotive MRF devices with excellent performance.

\section{Data Availability}

In this paper, the chain transformation process of magnetorheological fluids is analyzed from the micro point of view, so as to understand its rheological mechanism in depth. Through numerical simulation and experimental observation, the research is carried out. The numerical simulation is mainly simulated by MATLAB process programming (see Appendix for programming). The experimental observation is based on the static and dynamic platform designed by ourselves. Finally, the conclusion is drawn by comparing the numerical simulation and experimental observation. So the [DATA TYPE] data used to support the findings of this study are included in the article. The [DATA TYPE] data used to support the findings of this study are included in the supplementary information file.

\section{Conflicts of Interest}

The authors declare that they have no conflicts of interest.

\section{References}

[1] J. D. Carlson, "What makes a good MR fluid," Journal of Intelligent Material Systems and Structures, vol. 13, no. 7-8, pp. 431-435, 2002.

[2] X. Zhu, X. Jing, and L. Cheng, "Magnetorheological fluid dampers: a review on structure design and analysis," Journal of Intelligent Material Systems and Structures, vol. 23, no. 8, pp. 839-873, 2012.

[3] Z. P. Shulman, V. I. Kordonsky, E. A. Zaltsgendler, I. V. Prokhorov, B. M. Khusid, and S. A. Demchuk, "Structure, physical properties and dynamics of magnetorheological suspensions," International Journal of Multiphase Flow, vol. 12, no. 6, pp. 935-955, 1986.

[4] E. Lemaire, A. Meunier, G. Bossis et al., "Influence of the particle size on the rheology of magnetorheological fluids," Journal of Rheology, vol. 39, no. 5, pp. 1011-1020, 1995.

[5] X. Tang and H. Conrad, "An analytical model for magnetorheological fluids," Journal of Physics D: Applied Physics, vol. 33, no. 23, pp. 3026-3032, 2000.

[6] R. E. Rosensweig, "Suspensions de particules magnetiques: aspects du refroidissement," International Journal of Refrigeration, vol. 29, no. 8, pp. 1250-1258, 2006.

[7] X. Peng and H. Li, "Analysis of the magnetomechanical behavior of MRFs based on micromechanics incorporating a statistical approach," Smart Materials \& Structures, vol. 16, no. 6, pp. 2477-2485, 2007.

[8] C. Ciocanel, G. Lipscomb, and N. G. Naganathan, "A constitutive equation for magnetorheological fluid characterization," Journal of Phase Equilibria and Diffusion, vol. 29, no. 4, pp. 305-311, 2008.

[9] C. Yi, X. Peng, and C. Zhao, “A magnetic-dipoles-based micromacro constitutive model for MRFs subjected to shear deformation," Rheologica Acta, vol. 49, no. 8, pp. 815-825, 2010.

[10] W. Zhang, X. L. Gong, and L. Chen, "A Gaussian distribution model of anisotropic magnetorheological elastomers," Journal of Magnetism and Magnetic Materials, vol. 322, no. 23, pp. 3797-3801, 2010.

[11] W. Zhong, "Magnetic dipole and magnetic particle inspection: one of the principles of magnetic particle detection [J]," Nondestructive Testing, vol. 12, no. 3, pp. 66-70, 1990.

[12] S. Melle, O. G. Calderón, M. A. Rubio, and G. G. Fuller, "Rotational dynamics in dipolar colloidal suspensions: video microscopy experiments and simulations results," Journal of NonNewtonian Fluid Mechanics, vol. 102, no. 2, pp. 135-148, 2002.

[13] H. Abdi, R. Soheilian, R. M. Erb, and C. E. Maloney, "Paramagnetic colloids: chaotic routes to clusters and molecules," Physical Review E, vol. 97, no. 3, pp. 13-29, 2018.

[14] J. Winger, M. Schümann, A. Kupka, and S. Odenbach, "Influence of the particle size on the magnetorheological effect of magnetorheological elastomers," Journal of Magnetism and Magnetic Materials, vol. 481, no. 1, pp. 176-182, 2019.

[15] L. Yongzhi, L. Xinhua, and L. Hao, "The Monte Carlo simulation to magnetic particles of magnetorheological fluids," Procedia Engineering, vol. 15, pp. 3896-3900, 2011.

[16] D. H. Bae, W. J. Han, C. Y. Gao, Y. Z. Dong, and H. J. Choi, "Preparation and magnetorheological response of triangularshaped single-crystalline magnetite particle-based magnetic fluid," IEEE Transactions on Magnetics, vol. 54, no. 11, pp. 1-4, 2018. 
[17] M. Schümann and S. Odenbach, "In-situ observation of the particle microstructure of magnetorheological elastomers in presence of mechanical strain and magnetic fields," Journal of Magnetism and Magnetic Materials, vol. 441, no. 1, pp. 88-92, 2017.

[18] J. Rodríguez-López, P. Castro, L. Elvira, and F. M. de Espinosa, "Study of the effect of particle volume fraction on the microstructure of magnetorheological fluids using ultrasound: transition between the strong-link to the weak-link regimes," Ultrasonics, vol. 61, pp. 10-14, 2015.

[19] Q. Q. Wen, Y. Wang, and X. L. Gong, "The magnetic field dependent dynamic properties of magnetorheological elastomers based on hard magnetic particles," Smart Materials and Structures, vol. 26, no. 7, article 075012, 2017.

[20] P.-h. Zhao, Y.-z. Fu, H.-l. Li, C.-y. Zhang, and Y.-q. Liu, "Three-dimensional simulation study on the aggregation behavior and shear properties of magnetorheological fluid," Chemical Physics Letters, vol. 722, pp. 74-79, 2019.

[21] J. Xu, P. Wang, H. Pang et al., “The dynamic mechanical properties of magnetorheological plastomers under high strain rate," Composites Science and Technology, vol. 159, no. 5, pp. 50-58, 2018. 


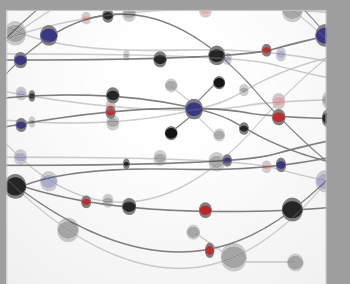

The Scientific World Journal
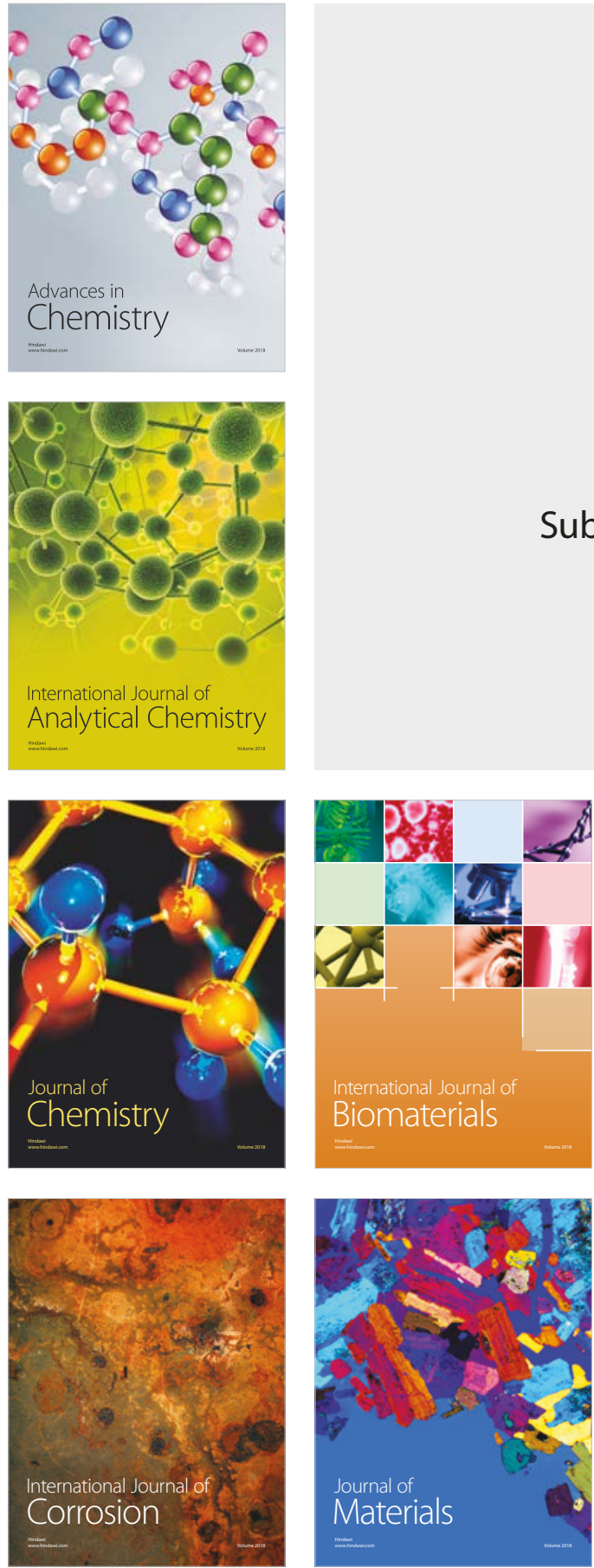

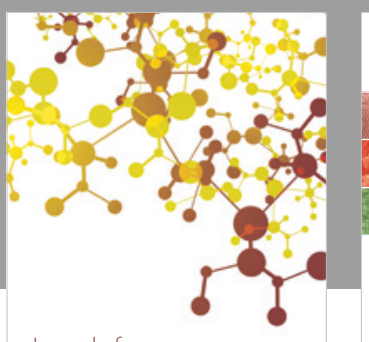

Journal of

Applied Chemistry
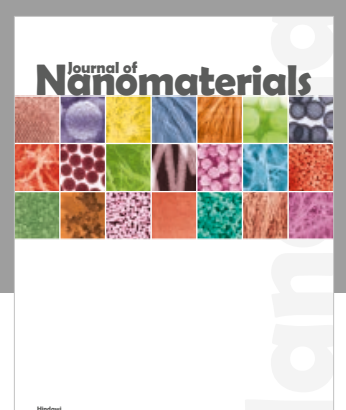

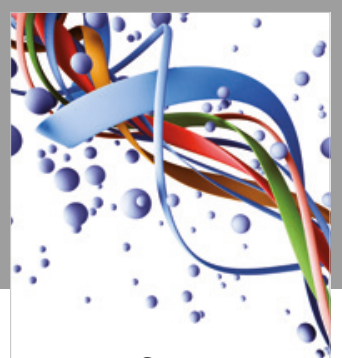

Scientifica

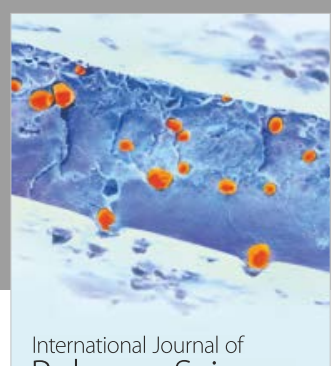

Polymer Science

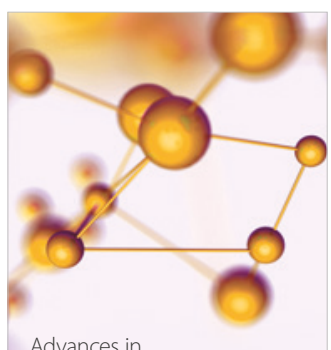

Physical Chemistry
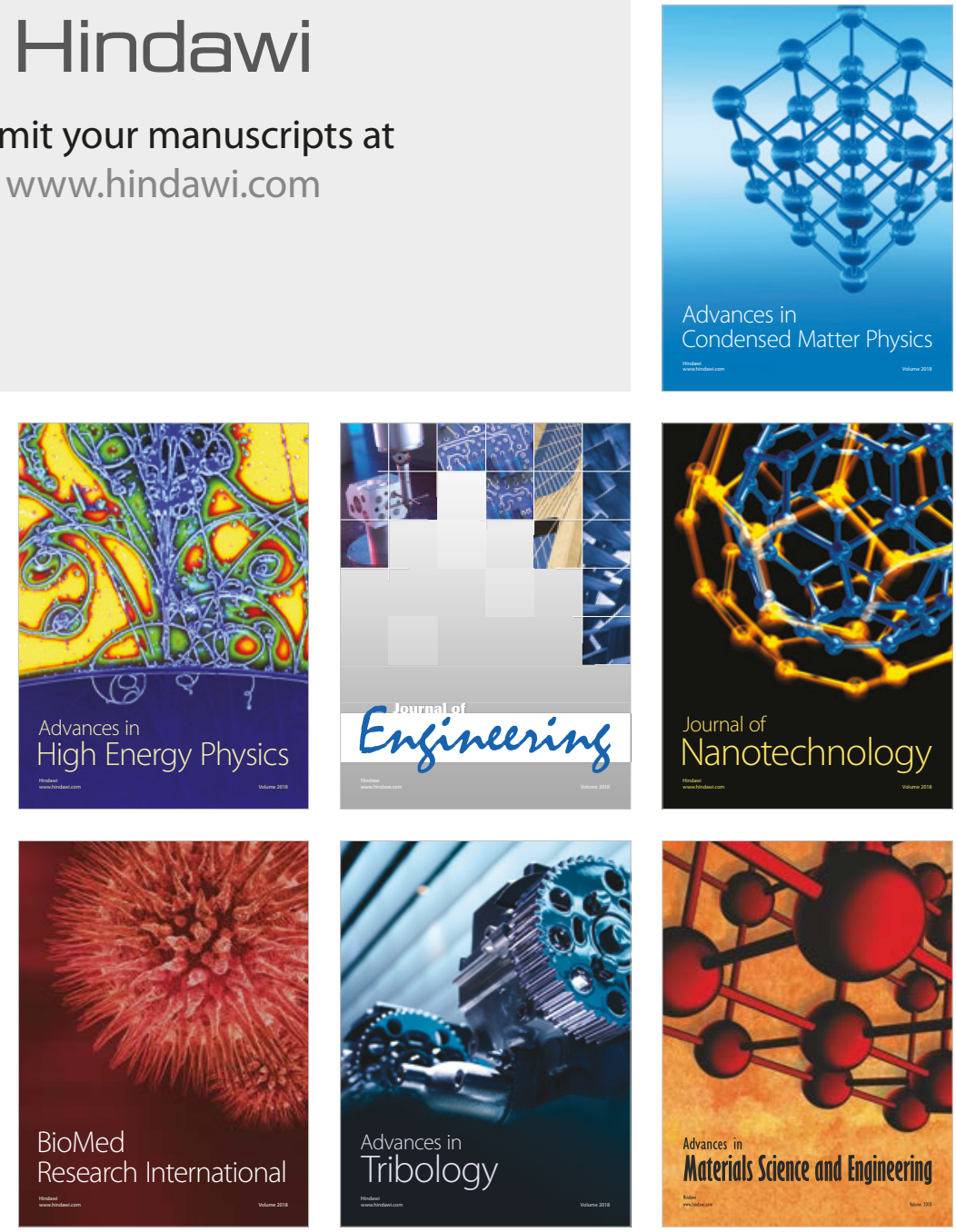